\title{
MODELS FOR THE OBSERVABLE SYSTEM PARAMETERS OF ULTRALUMINOUS X-RAY SOURCES
}

\author{
N. Madhusudhan, ${ }^{1}$ S. Rappaport, ${ }^{1}$ Ph. Podsiadlowski, ${ }^{2}$ and L. Nelson ${ }^{3}$ \\ Received 2007 October 5; accepted 2008 July 21
}

\begin{abstract}
We investigate the evolution of model populations of ultraluminous X-ray sources (ULXs), consisting of a black hole accretor in a binary with a donor star. Two of the models we consider invoke stellar-mass (up to $\sim 25 M_{\odot}$ ) black hole binaries (LMBHs), generated with a binary population synthesis code, while a third model uses intermediatemass $\left(\sim 1000 M_{\odot}\right)$ black hole accretors (IMBHs). For each model, we computed 30,000 binary evolution sequences. A scheme for calculating the optical flux from ULXs is discussed. We present "probability images" for the colormagnitude diagrams (CMDs) and for the orbital period-X-ray luminosity $\left(P_{\mathrm{orb}}-L_{\mathrm{x}}\right)$ plane. We show how a population of luminous X-ray sources in a cluster of stars evolves with time. The most probable ULX system parameters correspond to high-mass donors (of initial mass $\gtrsim 25 M_{\odot}$ ) with effective $\mathrm{O}$ through late $\mathrm{B}$ spectral types, and $P_{\text {orb }}$ between 1 and 10 days. Estimates of the numbers of ULXs in a typical galaxy as a function of $L_{\mathrm{x}}$ are also presented. We find that if LMBHs are allowed to have super-Eddington $L_{\mathrm{x}}$, the binding energy parameter for the stellar envelope of the black hole progenitor must be $\lambda \lesssim 0.03$ in order not to overproduce ULXs. Comparison of six known ULX counterparts with our model CMDs indicates that the IMBH model somewhat more closely matches the observations. We find that a significant contribution to the optical flux from the IMBH systems comes from intrinsic accretion disk radiation. In effect, IMBH systems, when operating at their maximum luminosities $\left(10^{41}-10^{42} \mathrm{ergs} \mathrm{s}^{-1}\right)$, are milli-AGNs. While models of IMBH systems during the X-ray phase are attractive, their formation mechanism remains uncertain.
\end{abstract}

Subject headings: accretion, accretion disks — binaries: general — black hole physics — galaxies: star clusters — X-rays: binaries

\section{INTRODUCTION}

An ultraluminous X-ray source (ULX) is an off-nucleus pointlike source whose X-ray luminosity, $L_{\mathrm{x}}$, exceeds $2 \times 10^{39} \mathrm{ergs} \mathrm{s}^{-1}$. An obvious candidate for such a source is an X-ray binary in which a compact object accretes from a donor star. Under normal circumstances, the accretion rate is constrained by the Eddington limit of the accretor. The Eddington limit for a neutron star is $\sim 10^{38} \mathrm{ergs} \mathrm{s}^{-1}$, and that for a $10 M_{\odot}$ black hole (BH) is $\sim 10^{39} \mathrm{ergs} \mathrm{s}^{-1}$. Therefore, if the accretors are assumed to be stellar mass BHs, the observed luminosities of ULXs exceed the Eddington limit by factors of up to $\sim 100$. The question as to how the Eddington limit could be violated remains a subject of considerable debate. On the other hand, if we assume the accretor to be an intermediate-mass black hole of $\sim 10^{2}-10^{4} M_{\odot}$, the ULX luminosities can all be accounted for without violating the Eddington limit. If the accreting stars are indeed IMBHs, the question remains as to how these objects are formed and how they acquire a massive stellar companion.

Several scenarios have been put forth to explain the apparent super-Eddington luminosities, assuming stellar-mass BHs. King et al. (2001) suggested that geometrical beaming in the direction of the observer, due to a thick accretion disk (Jaroszyński et al. 1980 ), could lead to both a high luminosity and an even higher inferred luminosity. Körding et al. (2002) make a similar argument, with relativistic beaming due to jets as the explanation. In both of these scenarios, the emission is considered to be anisotropic. These suggestions somewhat contradict the observations of ionization nebulae around some ULXs (Pakull \& Mirioni 2003), which tend to indicate the full implied isotropic luminosity. Another scenario,

\footnotetext{
${ }^{1}$ Department of Physics and Kavli Institute for Astrophysics and Space Research, Massachusetts Institute of Technology, Cambridge, MA 02139; nmadhu@mit.edu.

2 Department of Astrophysics, Oxford University, Oxford OX1 3RH, UK.

3 Physics Department, Bishop's University, Sherbrooke, QC J1M 0C8, Canada.
}

proposed by Begelman $(2002,2006)$, suggests that photon bubble instabilities in the accretion disk can lead to an isotropic luminosity exceeding the Eddington limit by a factor of $\sim 10$. Socrates \& Davis (2006) invoke a hot, optically thin corona in conjunction with a geometrically thin, but optically thick, accretion disk to explain the observed ULX luminosities. Yet other scenarios invoke "slim" accretion disks to produce approximately isotropic luminosities that may exceed the Eddington limit by up to factors of $\sim 10$ (Abramowicz et al. 1988; Ebisawa et al. 2003). Although these scenarios have not been shown to work conclusively, they could, in principle, account for ULX luminosities up to $\sim 10^{40} \mathrm{ergs} \mathrm{s}^{-1}$. In addition to these specific suggestions for bypassing the Eddington limit, the continuity and simplicity of the luminosity function of luminous X-ray sources from $10^{36}$ to $10^{40} \mathrm{ergs} \mathrm{s}^{-1}$ (a simple power law; Grimm et al. 2003) has led some to conclude that these represent a single class of systems, i.e., neutron stars and stellar-mass black holes (LMBHs) ${ }^{4}$ accreting from a normal donor star. However, we note that essentially none of these ideas involving stellar-mass BHs would plausibly be able to explain the ULXs at the higher end of the observed luminosities, namely, those with $L_{\mathrm{x}} \gtrsim 10^{40} \mathrm{ergs} \mathrm{s}^{-1}$.

By contrast, the IMBH scenario, as suggested first by Colbert \& Mushotzky (1999), accounts for the luminosities of all the ULXs because the Eddington limit for an IMBH in the mass range $\sim 10^{2}-10^{4} M_{\odot}$ varies between $10^{40}$ and $10^{42} \mathrm{ergs} \mathrm{s}^{-1}$. Other evidence that may suggest the presence of an IMBH includes observations of millihertz QPOs (Strohmayer \& Mushotzky 2003), ionization nebulae (Pakull \& Mirioni 2003), and inferences of cool inner accretion disk temperatures (Kaaret et al. 2003; Miller

\footnotetext{
${ }^{4}$ In this paper we refer to a stellar-mass black holes as "low-mass black holes" (LMBHs), rather than "SMBHs," which might be confused with "supermassive black holes."
} 
TABLE 1

Magnitudes and Colors of ULX Counterparts

\begin{tabular}{|c|c|c|c|c|}
\hline System & $M_{B}$ & $M_{V}$ & $B-V$ & $L_{\mathrm{x}}{ }^{\mathrm{a}}$ \\
\hline NGC 4559 X-7 (C1). & $-7.22 \pm 0.19$ & $-7.03 \pm 0.16$ & $-0.19 \pm 0.25$ & $\sim 10^{40}$ \\
\hline 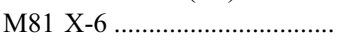 & $-4.28 \pm 0.04$ & $-4.18 \pm 0.03$ & $-0.10 \pm 0.05$ & $2 \times 10^{39}$ \\
\hline M101 ULX-1 ........... & $-6.19 \pm 0.15$ & $-5.92 \pm 0.12$ & $-0.27 \pm 0.19$ & $1.2 \times 10^{39}$ \\
\hline NGC 5408 ULX $^{\mathrm{b}}$. & $-6.40 \pm 0.20$ & $-6.40 \pm 0.20$ & $-0.00 \pm 0.28$ & $\sim 10^{40}$ \\
\hline Holmberg II ULX. & $-6.03 \pm 0.19$ & $-5.78 \pm 0.11$ & $-0.25 \pm 0.22$ & $\sim 10^{40}$ \\
\hline NGC 1313 X-2 (C1)................ & $-4.70 \pm 0.18$ & $-4.50 \pm 0.18$ & $-0.20 \pm 0.25$ & $\sim 10^{40}$ \\
\hline
\end{tabular}

Note.-Adapted from Copperwheat et al. (2007).

a Approximate X-ray luminosity in the $1-10 \mathrm{keV}$ band

${ }^{\mathrm{b}}$ Lang et al. (2007) have removed the ambiguity present in the optical identification in the Copperwheat et al. (2007) and Kaaret et al. (2003) papers.

et al. 2003, 2004; Cropper et al. 2004; but see Feng \& Kaaret 2007). Despite the several pieces of evidence supporting IMBH binaries as the model for ULXs, one of the main problems with this scenario lies in the unknown formation mechanism for such binaries. A few scenarios for IMBH formation have been proposed in the literature (see, for example, Portegies Zwart et al. 2004; Tutukov \& Fedorova 2005), but a definitive scenario remains elusive. In particular, the evolution of supermassive stars with $M \gg 100 M_{\odot}$ is not well understood.

In three of our earlier papers, we studied the evolution of populations of binary systems pertaining to both models for ULXs, namely: (1) super-Eddington accretion onto LMBHs (Podsiadlowski et al. 2003, Rappaport et al. 2005) and (2) sub-Eddington accretion onto IMBHs (Madhusudhan et al. 2006). In those studies, the main objective was to investigate the evolution of the X-ray luminosities of the systems with time, and to estimate the formation efficiencies for each scenario. In Podsiadlowski et al. (2003) and Rappaport et al. (2005), we considered LMBHs in binary systems with donor stars in the mass range $2-17 M_{\odot}$. Some of the binary populations were generated using the binary population synthesis (BPS) code developed in Podsiadlowski et al. (2003). There we showed that by allowing for a violation of the Eddington limit by a factor of $\sim 10-30$, such systems would be able to explain most of the observed ULXs, except for the very most luminous ones. In Madhusudhan et al. (2006) we considered donor stars in binary systems with IMBHs with a representative mass of $1000 M_{\odot}$. There we showed that in order to have a plausible formation efficiency for ULXs with $L_{\mathrm{x}} \gtrsim 10^{40} \mathrm{ergs} \mathrm{s}^{-1}$ the donor stars should be massive $\left(~ \gtrsim 8 M_{\odot}\right)$ and the initial orbital separations should be close $(\$ 6-40$ times the radius of the donor star when on the main sequence). Here we expand on this previous work, with emphasis on the predicted optical properties of ULXs.

A number of fairly secure optical identifications of ULX counterparts have now been reported in the literature. Liu et al. (2002) identified a unique counterpart to the ULX NGC 3031 X11 (in M81) and found it to be consistent with an O8 main-sequence (MS) donor star. Kaaret et al. (2004) reported an optical counterpart to the ULX in Holmberg II to be consistent with a donor star with spectral type between O4 V and B3 Ib. Kuntz et al. (2005) studied the optical counterpart of M101 ULX-1 and found the colors to be consistent with those for a mid-B supergiant. Soria et al. (2005) investigated the region surrounding the ULX in NGC 4559 and found that the brightest candidate counterpart within the Chandra error circle was a blue supergiant of high mass $\left(\sim 20 M_{\odot}\right)$. Mucciarelli et al. $(2005 ; 2007)$ found two candidate optical counterparts to the ULX NGC $1313 \mathrm{X}-2$ and reported the more likely candidate to be a B0-O9 main-sequence star. Finally, Kaaret et al. (2003) and Copperwheat et al. (2007) had tentatively identified a possible counterpart to the ULX in NGC 5408. Recently, Lang et al. (2007) uniquely identified the counterpart to this ULX, based on a radio detection; it turns out to be the same star as the one previous studies had identified as most likely. The counterpart has colors consistent with those for a B or early A supergiant.

The best candidate optical counterparts to the ULXs discussed above have been conveniently selected and summarized by Copperwheat et al. (2007). In particular, they tabulate the photometric values for the various systems, calculating reddening corrections and absolute magnitudes, wherever necessary. As can be seen from the above list, the observations seem to indicate that the spectral classes of the most promising candidates generally range between $\mathrm{O}$ and $\mathrm{B}$ spectral type, and the luminosity classes are either Vor Ib. In this study we consider optical counterparts of six ULX systems for which photometric data in the $B$ and $V$ bands are available in the literature. The photometric data for these systems are given in Table 1 (as adapted from Copperwheat et al. 2007).

Theoretical studies of ULXs in the optical regime have heretofore concentrated on constraining the nature of the donor star, the mass of the accretor, and the orbital period. All the models follow the standard paradigm of ULXs being X-ray binaries with active accretion through Roche-lobe overflow from a donor star onto a BH accretor. Rappaport et al. (2005) discussed preliminary theoretical models involving disk irradiation in ULXs. They presented sample evolution tracks, on a color-magnitude diagram (CMD), for four ULX models and discussed the optical appearance of ULX BH binaries. Pooley \& Rappaport (2005) suggested detection of X-ray and/or optical eclipses as a means to constrain the mass of the accretor. In recent studies, Copperwheat et al. (2005, 2007) have also constructed irradiation models to describe the optical emission from ULXs and used the models to constrain the properties of several systems observed in the optical. The parameters being considered were the mass, radius, and age of the donor, and the BH mass in some cases. From the fits to the various observations, they find the counterparts to be consistent with being main-sequence stars or evolved giants/supergiants with spectral types $\mathrm{O}, \mathrm{B}$, or $\mathrm{A}$.

In this paper we report a detailed population study spanning a large region of parameter space of ULX properties in an effort to better explore and help constrain ULX models. In particular, we investigate models of the optical properties of ULXs. We choose three sets of representative populations from our previous studies and explore their optical properties and other observables. For the models with LMBHs, we choose two sets of populations obtained from the BPS code corresponding to two different maximum primary masses. These two models yield distinctly different distributions of black hole masses. For models with IMBHs, we 
TABLE 2

ULX Population Models

\begin{tabular}{|c|c|c|c|c|c|}
\hline Model $^{\text {a }}$ & $M_{\mathrm{BH}}^{\mathrm{b}}$ & $M_{\mathrm{don}}^{\mathrm{c}}$ & $P_{\mathrm{orb}, i}{ }^{\mathrm{d}}$ & $\lambda \mathcal{E}_{\mathrm{CE}}^{\mathrm{e}}$ & $M_{\text {prim }}{ }^{\mathrm{f}}$ \\
\hline ................ & $6-15$ & $10-30$ & $1-12$ & 0.015 & $22-45$ \\
\hline $\mathrm{Lb}$ & $6-24$ & $10-34$ & $1-12$ & 0.015 & $22-65$ \\
\hline Ic & 1000 & $5-50$ & $1-10$ & $\ldots$ & $\ldots$ \\
\hline
\end{tabular}

${ }^{\text {a }}$ The distributions of masses and orbital periods for the different models are shown in Fig. 1.

${ }^{\mathrm{b}}$ Approximate range of black-hole masses, in units of $M_{\odot}$.

c Approximate range of donor masses, in units of $M_{\odot}$.

d Approximate range of initial orbital periods, in units of days.

e Dimensionless inverse binding energy of the envelope of the black hole progenitor star, $\lambda$, multiplied by the dimensionless energy efficiency for ejecting the common envelope, $\mathcal{E}_{\mathrm{CE}}$. These quantities are defined in eq. (2) in the text.

${ }^{\mathrm{f}}$ Approximate range of primary star masses, in units of $M_{\odot}$.

choose model C from Madhusudhan et al. (2006). This model yielded the highest formation efficiency for ULXs in the IMBH scenario. For each of the three populations, we follow 30,000 binary evolution calculations. The models are summarized in Table 2.

The X-ray luminosities are calculated using a standard formulation of Roche-lobe mass transfer in X-ray binaries. The optical flux from the system is determined as the sum total of the optical flux from the donor star, X-ray irradiation of the accretion disk, and intrinsic energy generation in the disk. We present detailed CMDs for all the models. These include CMDs for the binary system, i.e., the sum of the optical flux of the donor and that due to radiation from the disk, as well as CMDs for the donor star alone. We also study in detail the evolution of the X-ray luminosity with the age of the system, $t_{\mathrm{ev}}$, and the evolution of the X-ray luminosity with orbital period, $P_{\text {orb }}$.

\section{METHODS}

\subsection{Binary Population Synthesis}

In our previous studies we explored several models of ULX populations for both the LMBH and IMBH scenarios (Rappaport et al. 2005; Madhusudhan et al. 2006). In this work we chose two LMBH binary populations and one IMBH binary population that were motivated by these previous studies. The parameters for the three models are summarized in Table 2, and the distributions of the initial system masses and orbital periods are shown in Figure 1. Models $\mathrm{La}$ and $\mathrm{Lb}$ are LMBH populations, whereas model Ic represents an IMBH population.

To generate the LMBH binary populations, we used the binary population synthesis (BPS) code developed in Podsiadlowski et al. (2003). We briefly review here the formulation from Rappaport et al. (2005). We started with a very large set of massive primordial binaries and generated a much smaller subset of these that evolved to contain a black hole and a relatively unevolved companion star. The product was a set of "incipient" black hole X-ray binaries with a particular distribution of orbital periods, $P_{\text {orb }}$, donor masses, $M_{\text {don }}$, and black hole masses, $M_{\mathrm{BH}}$, for each of a number of different sets of input assumptions (see, e.g., Fig. 2 of Podsiadlowski et al. 2003). For this part of the calculation, we employed various "prescriptions," based on single-star evolution models for the primary, simple orbital dynamics associated with wind mass loss and transfer, assumptions about the magnitude of the wind mass loss from the primary as well as from the core of the primary after a common-envelope phase, and natal kicks during the core collapse and formation of the black hole. After the common envelope has ejected the envelope of the primary, we take the subsequent wind mass loss from the core, before it collapses to form a black
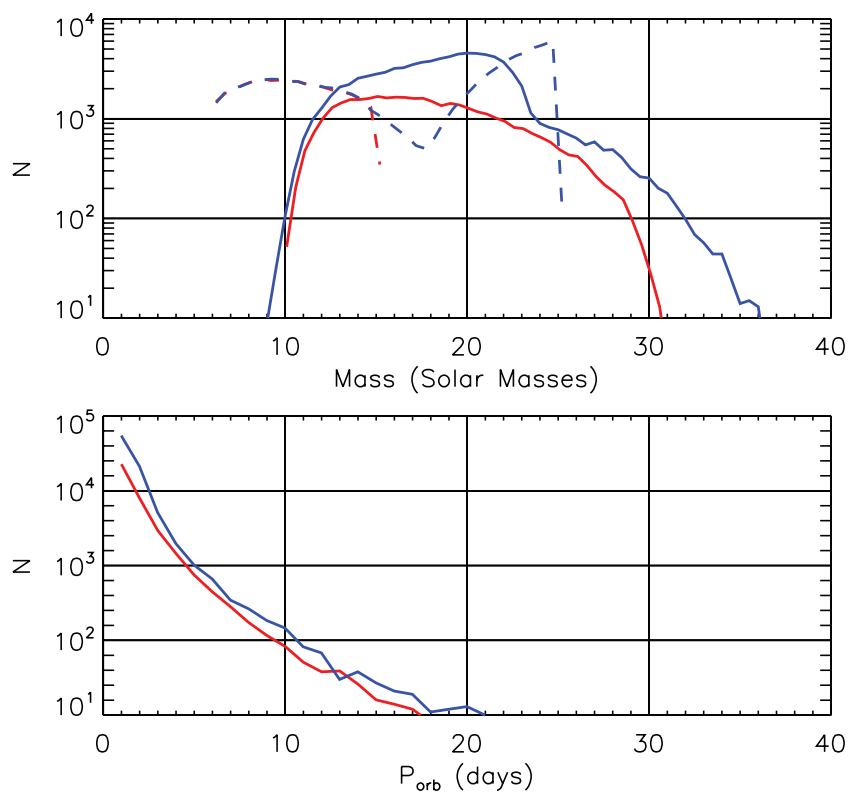

FIG. 1.-Distributions of initial donor masses, black hole masses, and initial orbital periods of the LMBH systems. The red curves correspond to model La, and the blue curves to model $\mathrm{Lb}$. In the top panel the solid curves represent donor masses and the dashed curves represent BH masses. For model Ic, $M_{\mathrm{BH}}=1000 M_{\odot}$ and we choose $M_{\text {don }}$ uniformly between 5 and $50 M_{\odot}$, and the initial orbital separation uniformly between 1 and 3 times the separation required for a ZAMS star of the corresponding donor mass to fill its Roche lobe.

hole, to be $\sim 25 \%$ of the core mass. Specifically, we take the black hole mass to be $75 \%$ of the core mass of the primary star, i.e., $M_{\mathrm{BH}}=0.75 \times\left(0.12 M_{\text {prim }}^{1.35}\right)$, where $M_{\text {prim }}$ is the mass of the primary. We further assume that there are no significant natal kicks associated with the collapse leading to the formation of the black holes.

The decision in the binary population synthesis code of whether or not a common-envelope phase occurs, when the primary first overflows its Roche lobe and starts to transfer mass onto the secondary, is based on the evolutionary state of the primary when mass transfer commences. We first define $R_{\text {TAMS }}$ and $R_{\mathrm{HG}}$ as the radii of the primary when it reaches the terminal-age main sequence (TAMS) and the end of the Hertzsprung gap, respectively. Given the initial orbital separation and the mass ratio of the primordial binary, $q \equiv M_{\text {prim }} / M_{\text {sec }}$, we can compute the Roche-lobe radius, $R_{L}$, of the primary. In order to form a relatively close black hole binary of the type we are considering in this work (i.e., $P_{\text {orb }} \lesssim 15$ days), we require that the initial mass transfer rate from the primary to the secondary be so large that it leads to a common-envelope and spiral-in phase. This depends mainly on the initial mass ratio and the envelope structure of the mass donor. If the donor star has a convective envelope, mass transfer tends to be dynamically unstable if the mass of the donor star significantly exceeds the mass of the secondary. The critical mass ratio in this case depends on the mass of the star and the mass loss from the system; detailed binary evolution calculations show that the critical mass ratio is typically in the range of 1.1 to 1.3 (e.g., Podsiadlowski et al. 2002; Han et al. 2002; Maund et al. 2004); in this study we adopt a value of 1.2. If the donor star has a radiative envelope, then mass transfer is dynamically stable for mass ratios as large as 3 to 4 (e.g., Han et al. 2000; Podsiadlowski et al. 2002). However, even if mass transfer is dynamically stable, the mass transfer rate may be so large that the accretor swells up and the system forms a commonenvelope configuration (a contact binary; Pols 1994; Wellstein et al. 2001). Since the mass in the common envelope in this case 
is very small, it is presently unclear whether this will lead to a spiral-in phase or just a transient contact configuration. Here we adopt what we consider a reasonable, albeit highly uncertain, critical mass ratio of 2 , above which systems in the Hertzsprung gap experience a spiral-in phase. Hence, we use the following critical mass ratios, $q_{\text {crit }}$, depending on the relation among $R_{L}$, $R_{\mathrm{TAMS}}$, and $R_{\mathrm{HG}}$ :

$$
q_{\text {crit }}= \begin{cases}2, & \text { for } R_{\mathrm{TAMS}}<R_{L}<R_{\mathrm{HG}} \\ 1.2, & \text { for } R_{L}>R_{\mathrm{HG}}\end{cases}
$$

Simple energetic arguments were used to yield the final-toinitial orbital separation during the common-envelope phase wherein the envelope of the primary is ejected. Here we used a parameter $\lambda$, which is the inverse of the binding energy of the primary envelope at the onset of the common-envelope phase in units of $G M_{\text {prim }} M_{e} / R_{\text {prim }}$, where $M_{\text {prim }}, M_{e}$, and $R_{\text {prim }}$ are the total mass, envelope mass, and radius of the primary, respectively. This parameter strongly affects the final orbital separation after the commonenvelope phase, where smaller values of $\lambda$ correspond to more tightly bound envelopes, and hence more compact post-commonenvelope orbits.

With the above parameterization for the ejection of the common envelope we find the following expression for initial-final orbital separation (e.g., Webbink 1985; Dewi \& Tauris 2000; Pfahl et al. 2003):

$$
\left(\frac{a_{f}}{a_{i}}\right)_{\mathrm{CE}} \simeq \frac{M_{c} M_{\mathrm{sec}}}{M_{\mathrm{prim}}}\left(M_{\mathrm{sec}}+\frac{2 M_{e}}{\mathcal{E}_{\mathrm{CE}} \lambda \mathcal{R}_{L}}\right)^{-1},
$$

where the subscripts prim, $c$, and $e$ stand for the progenitor of the black hole, its core, and its envelope, respectively, and "sec" is for the secondary, i.e., the progenitor of the "donor star" in the black hole binary. The quantity $r_{L}$ is the Roche-lobe radius of the black hole progenitor in units of $a_{i}, \mathcal{E}_{\mathrm{CE}}$ is the fraction of the gravitational binding energy between the secondary and the core of the black-hole progenitor that is used to eject the common envelope, and $\lambda$ is defined above. For typically adopted parameter values, $\lambda \sim 0.01-1$ (e.g., Dewi \& Tauris 2000; Podsiadlowski et al. 2003), $\mathcal{E}_{\mathrm{CE}} \simeq 1$ and $r_{L} \simeq 0.45-0.6$ (for an assumed mass ratio between the black hole progenitor and the companion in the range $\sim 2: 1 \rightarrow 15: 1$ ), the second term within the parentheses in equation (2) dominates over the first. In this case, we find the following simplified expression for $a_{f} / a_{i}$ :

$$
\left(\frac{a_{f}}{a_{i}}\right)_{\mathrm{CE}} \simeq \frac{r_{L}}{2}\left(\frac{M_{c}}{M_{\mathrm{prim}} M_{e}}\right) M_{\mathrm{sec}} \lambda \simeq 0.005\left(\frac{M_{\mathrm{sec}}}{M_{\odot}}\right) \lambda,
$$

where the leading factor is $r_{L} / 2 \simeq 1 / 4$, while the factor in parentheses involving the black hole progenitor is $\sim 0.020 \pm 0.002 M_{\odot}^{-1}$ for virtually all of the progenitors we consider. This explains why the large majority of the incipient black hole binaries (with lowto intermediate-mass donors) found by Podsiadlowski et al. (2003) resulted from an initially very wide orbit $\left(P_{\text {orb }} \sim\right.$ years - when the primary attains radii of $\sim 1000-2300 R_{\odot}$ ) preceding the commonenvelope phase in order to avoid a merger between the secondary and the core of the primary. ${ }^{5}$

\footnotetext{
5 We note that this formalism may not be applicable if the CE phase starts early in the Hertzsprung gap, i.e., soon after the primary has left the main sequence. In this phase, the primary has not yet developed a well-defined core-envelope structure, and a complete merger may always be unavoidable (e.g., Belczynski et al. 2007). However, we find from our simulations that most of our successful systems (i.e., those that experience a common-envelope phase and avoid merging) commence mass transfer either near the end of the Hertzsprung gap or beyond and therefore have well-defined core-envelope structures.
}

In this work, for models La and Lb (see Table 2 and Fig. 1), we chose two populations that were obtained using the BPS code described above. These two LMBH populations both use an inverse common-envelope binding energy parameter $\lambda=0.03$ and a common-envelope ejection efficiency factor of $\mathcal{E}_{\mathrm{CE}}=0.5$ (see eq. [2]; see, e.g., Dewi \& Tauris 2000; Podsiadlowski et al. 2003). The distinction between the two LMBH models lies in the upper mass limit adopted for the black-hole progenitor stars: 45 vs. $65 M_{\odot}$. The black holes in the model with lower mass primaries are constrained to $\lesssim 15 M_{\odot}$, while the higher mass primaries yield black holes up to $\sim 24 M_{\odot}$ (see, e.g., Prestwich et al. 2007). Finally, we take the lower limit on the mass of the primary, in order to form a black hole, to be $\gtrsim 24 M_{\odot}$, although this value depends on the equation of state of neutron star matter (and hence the upper mass limit for neutron stars).

For the IMBH case, model Ic (see Table 2), we chose the same population as model C in Madhusudhan et al. (2006). Due to our lack of a firm understanding of how IMBHs form in star clusters, and how they capture companion stars, we adopted a very simple prescription for the population of incipient IMBH binaries (but see Blecha et al. 2006). For each binary, we chose the initial donor mass to lie uniformly in the range of 5-50 $M_{\odot}$ using Monte Carlo methods. And we chose the initial orbital separation uniformly over the range of 1-3 times the separation required for a ZAMS star of the corresponding mass to fill its Roche lobe. This translates to a range of initial orbital separations between 40 and $200 R_{\odot}$, and $P_{\text {orb }}$ in the range $\sim 1-10$ days. We found in our previous work that this population is the most favorable one we explored for producing ULXs in the IMBH scenario, i.e., the donor stars should be massive, i.e., $\gtrsim 8 M_{\odot}$ and the initial orbital separations, after circularization, should be close, $\sim 6-40$ times the radius of the donor star when on the ZAMS. This range of close orbital separations represents the case of direct tidal capture and circularization of a single field star by the IMBH (see, e.g., Hopman et al. 2004; Pfahl 2005).

For each of the three modeled ULX populations, we carried out 30,000 binary evolution calculations. All models were run using 60 nodes of the elix3 Beowulf cluster located at the University of Sherbrooke, Quebec. The run time for each of the three models was $\sim 40 \mathrm{hr}$.

\subsection{Stellar Evolution}

The stellar evolution of the donor stars, including mass loss, was followed with EZ which is a stripped down, rewritten version of a subset of the stellar evolution code developed by P. P. Eggleton (Paxton 2004). The physics of the program is unchanged from Eggleton's (essentially as described in Pols et al. 1995), but the structure of the code has been modified to facilitate experiments involving programmed control of parameters. There are zero-age main-sequence (ZAMS) starting models for a variety of metallicities (from $Z=10^{-4}$ to 0.03 ) and masses (from 0.1 to $100 M_{\odot}$ ), with arbitrary starting masses created by interpolation. A userprovided procedure is called between steps of the evolution to inspect the current state, to make changes in parameters, and to decide when and what to record to $\log$ files. ${ }^{6}$ For all models in this particular study, the number of stellar mesh points was fixed at 200 in the interest of minimizing computation time.

\subsection{Binary Evolution Calculations}

The binary evolution was governed by a sequence of calculations involving the mass transfer rate, the corresponding change

\footnotetext{
6 The source code and data for EZ can be downloaded from the Web at http:// theory.kitp.ucsb.edu/ paxton.
} 
in orbital separation, and the subsequent monitoring of mass loss. The mass transfer considered was due solely to Roche-lobe overflow. When the donor star fills its Roche lobe, the excess matter flows through the inner Lagrange point onto the accretor. Assuming spherical geometry, the mass-transfer rate was calculated using $\dot{M} \simeq 2 \pi R H \rho v$, where $R$ is the radius of the donor, $H$ is the density scale height of the donor atmosphere, $\rho$ is the density of the atmosphere at the Roche lobe, and $v$ is the thermal velocity at the photosphere. Under the approximation of an isothermal, constantgravity atmosphere, the scale height is given by $H \simeq k T / \mu g$, where $\mu$ is the mean molecular weight, and the density profile of the atmosphere is exponential. The Roche-lobe radius of the donor was taken to be $R_{L}=0.49 a q^{2 / 3}\left[0.6 q^{2 / 3}+\ln \left(1+q^{1 / 3}\right)\right]^{-1}$ (Eggleton 1983 ), where $a$ is the separation of the binary, $q=M_{\mathrm{don}} / M_{\mathrm{BH}}, M_{\mathrm{don}}$ is the mass of the donor, and $M_{\mathrm{BH}}$ is the mass of the black hole. This semi-explicit procedure, while making use of some approximations, is self-adjusting to yield the same mass transfer rates that would be obtained from more explicit calculations of $\dot{M}$.

The Eddington limited mass transfer rate onto the accretor is given by $\dot{M}_{\text {Edd }}=4 \pi G M_{\text {acc }} / \eta \kappa c$, where $G$ is the gravitational constant, $\kappa$ is the radiative opacity, and $\eta$ is the efficiency of the black hole accretor in converting rest mass to radiant energy. The opacity is assumed to be predominantly due to electron scattering and is given by $\kappa=0.2(1+X) \mathrm{cm}^{2} \mathrm{~g}^{-1}$, where $X$ is the hydrogen mass fraction. For a spinning black hole, we take the radiation efficiency to be given by $\eta=1-\left[1-\left(M / 3 M_{0}\right)\right]^{1 / 2}$, where $M_{0}$ is the initial mass of the black hole (Bardeen 1970). This assumes an initially nonspinning black hole.

If we consider all the matter leaving the donor to be retained by the accretor, independent of the Eddington limit, then the resulting luminosity is referred to as the "potential luminosity" and is given by $L_{\text {pot }}=\eta \dot{M} c^{2}$. The actual isotropic X-ray luminosity, on the other hand, is given by $L_{\mathrm{x}}=\beta \eta \dot{M} c^{2}$, where $\beta$ restricts the mass-transfer rate to the Eddington limit. For $\dot{M}<\dot{M}_{\mathrm{Edd}}, \beta=1$, otherwise $\beta=\dot{M}_{\text {Edd }} / \dot{M}$. When $\dot{M}>\dot{M}_{\text {Edd }}$, it is assumed that all the matter passes through the accretion disk until it reaches the inner edge of the disk, at which point the excess matter (above the Eddington limit) is ejected out of the system in the form of a jet. The mass and orbital angular momentum lost from the system in such ejection is incorporated when calculating the orbital separation of the system. We emphasize that we use the Eddington limited mass transfer rate while calculating the orbital parameters during the evolution of the binary. However, along with the various orbital parameters, we also record $L_{\text {pot }}$ at each step. And in all the results presented in this paper we typically refer to $L_{\text {pot }}$ instead of the X-ray luminosity, $L_{\mathrm{x}}$, of the system in order to examine the potentialities of violating the Eddington limit.

\subsection{Disk Irradiation}

The optical flux from a ULX is comprised of contributions from the donor star and from the reprocessing of X-ray photons by the accretion disk (as well as intrinsic energy generation within the disk). The irradiation of the accretion disk depends on $L_{\mathrm{x}}$, the geometrical properties of the disk, and whether or not the central accreting star has a hard surface. For purposes of the current calculation, we assume the disk to be geometrically thin. We find the effective temperature of the disk to be

$$
T(r) \simeq\left(\frac{L_{\mathrm{x}}}{4 \pi r_{\min }^{2} \sigma}\right)^{1 / 4}\left[\frac{4}{7} \xi^{\prime 2} x^{-10 / 7}(1-\alpha)+3 x^{-3}\right]^{1 / 4}
$$

Here $x=r / r_{\min }$, where $r$ is the radial distance, $r_{\min }$ is the inner disk radius, taken to be $6 G M_{\mathrm{BH}} / c^{2}, \alpha$ is the X-ray albedo of the disk, which we take to be 0.7 . The half thickness of the disk is given by $h(r)=\xi r^{9 / 7} r_{\max }^{-2 / 7}$ and $\xi^{\prime}=\xi\left(r_{\min } / r_{\max }\right)^{2 / 7}$, where $\xi$ is a constant equal to $h\left(r_{\max }\right) / r_{\max }$ and $r_{\max }$ is the outer radius of the disk. We have taken $r_{\max }$ to be $0.7 r_{L} a$ where $r_{L}$ is the dimensionless Roche-lobe radius of the black hole accretor. We somewhat arbitrarily adopt a value for $\xi$ of 0.1 , corresponding to a full angular thickness of the disk of $\sim 12^{\circ}$. The second term inside the square brackets in equation (4) comes from the Shakura \& Sunyaev (1973) solutions for the temperature profile of a thin disk around a BH accretor. We have taken the inner edge of the accretion disk to be at the innermost stable circular orbit around a nonrotating $\mathrm{BH}$. We have neglected the factor $\left[1-\left(r_{\min } / r\right)^{1 / 2}\right]$, since its contribution to the optical flux can be assumed to be negligible. The first term inside the square brackets in equation (4) is adapted from the expression for the irradiation temperature of an accretion disk around a BH derived in King et al. (1997). This is a modified version of the corresponding term in equation (8) of Rappaport et al. (2005), where we used an expression appropriate for radiation emanating from a centrally located, isotropically emitting, hard surface. The present formulation takes into account the fact that the X-ray radiation comes from the innermost part of the accretion disk surface and is emitted with a Lambertian angular distribution. Since the irradiated portion of the outer disk lies at a shallow angle with respect to the surface of the inner disk, the small value of the cosine factor significantly reduces the X-ray irradiation.

As an important caveat to our disk irradiation calculations, we note that the assumption of a thin disk (emitting in a Lambertian manner) likely breaks down at accretion rates slightly or greatly exceeding Eddington. In this case, the irradiation of the outer disk might become more or less efficient. However, such a calculation is considerably beyond the scope of the present work. Notwithstanding the complexity of the full problem of evaluating the optical reprocessing from a thick inner disk, in $\S 3.5$ we present results for an alternate irradiation prescription that could mock up more efficient heating for the thick-disk case.

The optical flux from the disk is determined by using the temperature profile described in equation (4) and assumes that the local spectrum in each annulus of the disk is thermal. The radiant flux from each annular ring on the disk surface is determined from the specific intensity of the blackbody radiation and the temperature at that radius. The total flux due to the disk at a particular wavelength is calculated by integrating the annular flux over the radial extent of the disk, from $r_{\min }$ to $r_{\max }$. The flux from the donor star is determined from its surface temperature, $T_{e}$, as obtained from the stellar evolution code. For the wavelengths under consideration, a thermal blackbody is a reasonable approximation in order to determine the flux from the star (with $\sim 10 \%$ accuracy). This corresponds to an error in the apparent $V$ magnitude of $\sim \pm 0.1$, which is within the error bars of most observations, and is negligible compared to the absolute values of $V$ and $M_{V}$. The corresponding error in $B-V$ is $\lesssim 0.2$.

The total flux of the system consists of contributions from the disk and the donor star. The $B$ and $V$ magnitudes were calculated from the total fluxes using $B=-2.5 \log \left(F_{B} / F_{B 0}\right)$ and $V=$ $-2.5 \log \left(F_{V} / F_{V 0}\right)$, where $F_{B}, F_{B 0}, F_{V}$, and $F_{V 0}$ are the fluxes and reference fluxes at $\lambda=4380 \AA$ and $\lambda=5450 \AA$, respectively $\left(F_{B 0}=6.61 \times 10^{-9} \operatorname{ergs~cm}^{-2} \mathrm{~s}^{-1} \AA^{-1}\right.$ and $F_{V 0}=3.64 \times$ $\left.10^{-9} \operatorname{ergs~cm} \mathrm{cm}^{-2} \mathrm{~s}^{-1} \AA^{-1}\right)$.

After we compute $B-V$ for the combined radiation from the donor star and the accretion disk, ${ }^{7}$ we apply a small correction

\footnotetext{
7 All of our binary systems are assumed to be viewed at an average orbital inclination angle of $60^{\circ}$.
} 


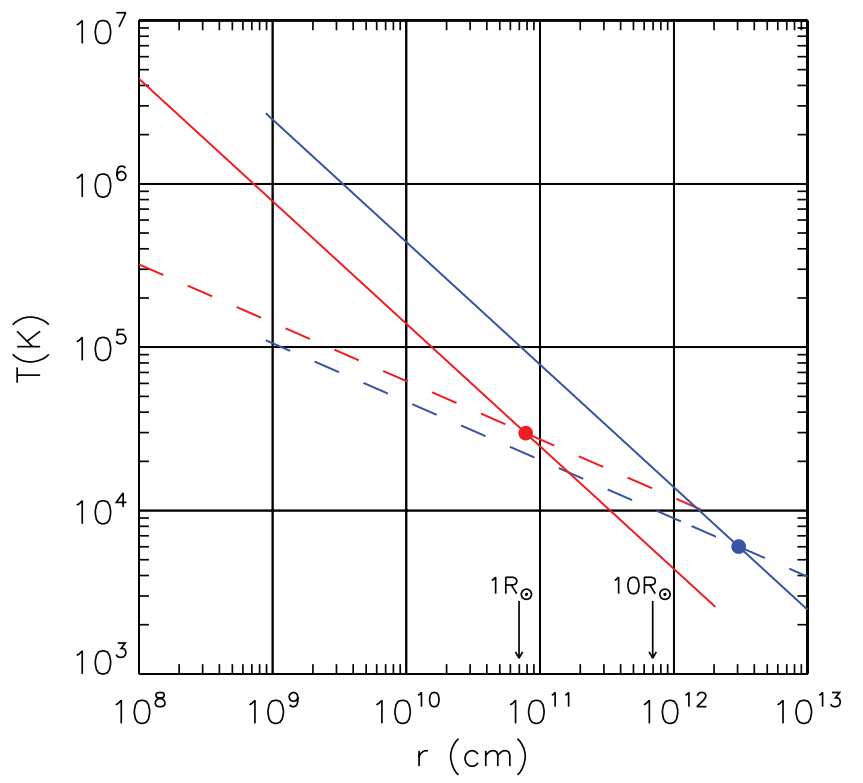

FIG. 2.- Illustrative temperature profiles of the irradiated accretion disk for an IMBH model (blue curves) and LMBH model (red curves). The solid curves are for the case of intrinsic (viscous) heating of the accretion disk alone, while the dashed curves are for the irradiation of the disk without any contributions from viscous heating. These correspond to the second and the first terms, respectively, inside the square brackets of eq. (4). The binary system parameters used for this illustrative example are given in the text.

( $\lesssim 0.2 \mathrm{mag}$ ) as a function of $B-V$ to take into account the treatment of the radiation as blackbody emission. These corrections were based on the differences between the $B-V$ colors computed from the simple algorithm given above and the colors of mainsequence and supergiant stars tabulated by Johnson (1966).

\subsection{Intrinsic Disk Emission}

We have found from our models (see $\S 3$ ) that under certain circumstances the intrinsic disk emission (due to the viscous release of gravitational potential energy), which is represented by the second term inside square brackets in equation (4), can dominate the optical emission from the disk irradiation (first term inside square brackets). To illustrate this effect, we show in Figure 2 the temperature profiles for an illustrative set of binary parameters: $L_{\text {pot }}=10^{40} \mathrm{ergs} \mathrm{s}^{-1}, P_{\text {orb }}=30$ days and $M_{\text {don }}=10 M_{\odot}$. For an IMBH model $\left(M_{\mathrm{BH}}=1000 M_{\odot}\right)$ the minimum and maximum disk radii are $r_{\text {min }}=9 \times 10^{8} \mathrm{~cm}$ and $r_{\text {max }}=1.5 \times 10^{13} \mathrm{~cm}$, respectively, while for an LMBH model (with $M_{\mathrm{BH}}=10 M_{\odot}$ ), these radii are $r_{\min }=9 \times 10^{6} \mathrm{~cm}$ and $r_{\max }=2 \times 10^{12} \mathrm{~cm}$.

The red and blue curves in Figure 2 are for the LMBH and IMBH models, respectively. The dashed curves are the temperature profiles for the case where irradiation is the only source of energy input to the disk; conversely, the solid curves are for the intrinsic (viscous) release of gravitational energy alone - without X-ray irradiation. For the LMBH model, we see that the intrinsic energy release dominates the irradiation contribution to the optical radiation only for radial distances in the disk of $\lesssim 1 R_{\odot}$. Since the optical light from the donor star itself comes from a typically much larger area, the intrinsic disk emission is generally not competitive with that from disk irradiation in the size and temperature ranges that matter. By contrast the crossing point for the two temperature profiles corresponding to the IMBH model occurs at a radial distance of $\sim 55 R_{\odot}$ [and $T(r)$ remains $\gtrsim 10^{4} \mathrm{~K}$ out to $\gtrsim 20 R_{\odot}$ ]. These radii are larger than the size of the donor star while it is near the main sequence or subgiant branch (i.e., while it is hot). Thus, it is quite possible that the largest contribution to the optical light from the IMBH systems comes from the intrinsic radiation emitted by the accretion disk itself. We will see the effect of this in $\S 3$ (see especially Fig. 6).

\subsection{Irradiation of the Donor Star}

We do not consider the effects of X-ray irradiation of the donor star. For our IMBH models the half angle subtended by the donor star ranges between $\sim 5^{\circ}$ and $10^{\circ}$. Since we have assumed an accretion disk with only a modest half angle of $6^{\circ}$, most or all of the donor star can be expected to be shielded by the accretion disk. For the LMBH models the donor stars, at least initially, subtend larger half angles of $\sim 18^{\circ}-24^{\circ}$. The fractional solid angle that the donors subtend ranges between $\sim 0.025$ and 0.036 while they are on the main sequence (and even less when they are on the giant branch and have transferred a significant amount of their mass). After taking into account shadowing by the accretion disk, the albedo of the donor star surface, and the Lambertian angular dependence of the disk irradiator, we find that no more than $0.0018 \rightarrow 0.0026$ of the X-ray luminosity is reprocessed on the face of the donor star. This is less than comes from the irradiated accretion disk, and, furthermore, the larger distance between the $\mathrm{X}$-ray source and the donor compared to that of the irradiated disk renders the flux even smaller yet. Therefore, for this work, we have neglected the irradiation of the donor stars.

\section{RESULTS AND DISCUSSION}

We present models of ultraluminous X-ray sources including calculations of the optical flux from the system. The optical flux includes contributions from the donor star as well those due to radiation from the accretion disk. As mentioned previously, we have chosen three representative populations of binary systems for our study. Two of these populations have LMBHs and one population has IMBHs as accretors. At the beginning of each evolution, the donor star is on the zero-age main sequence (ZAMS), and the initial system parameters are set by the binary population synthesis algorithms described above.

\subsection{Evolution of a Single Binary}

Figure 3 shows sample evolutionary tracks for three individual ULX binaries. The three binaries are illustrative of the three models listed in Table 2. Starting from the top left: panel $a$ shows the evolution of $L_{\text {pot }}$ with the age of the system $\left(t_{\mathrm{ev}}\right)$. Panel $b$ presents the evolution of $L_{\text {pot }}$ with the orbital period, $P_{\text {orb }}$. Panel $c$ shows the track in the CMD of the ULX binary, taking into account the optical flux from the donor star as well as the flux due to radiation from the disk. And, panel $d$ represents the CMD of the donor star alone. In calculating the optical flux from the disk, $L_{\text {pot }}$ was used to irradiate the disk, which allows for possible violation of the Eddington limit. The evolution of the various parameters with time is denoted by the arrows. The green curves represent the evolution of an IMBH binary with an initial donor mass, $M_{\text {don }}=$ $30 M_{\odot}$, a black hole mass, $M_{\mathrm{BH}}=1000 M_{\odot}$, and an initial orbital period, $P_{\text {orb }}=2.1$ days; taken from model Ic (see Table 2). The blue curves represent model Lb with $M_{\mathrm{don}}=14 M_{\odot}, M_{\mathrm{BH}}=23 M_{\odot}$, and $P_{\text {orb }}=1.7$ days. And, the red curves are for model La with $M_{\text {don }}=7 M_{\odot}, M_{\mathrm{BH}}=11 M_{\odot}$, and $P_{\text {orb }}=1.6$ days. In order to illustrate the direction of evolution of the tracks, on each panel arrows are marked on one of the tracks.

Let us consider the $L_{\text {pot }}-t_{\mathrm{ev}}$ tracks in panel $a$ for illustration, and follow the blue curve. The evolution starts with the donor on the ZAMS. As the donor evolves through the main sequence and somewhat beyond, the radius increases slightly and the donor fills its Roche lobe. Mass transfer then takes place onto the accretor 

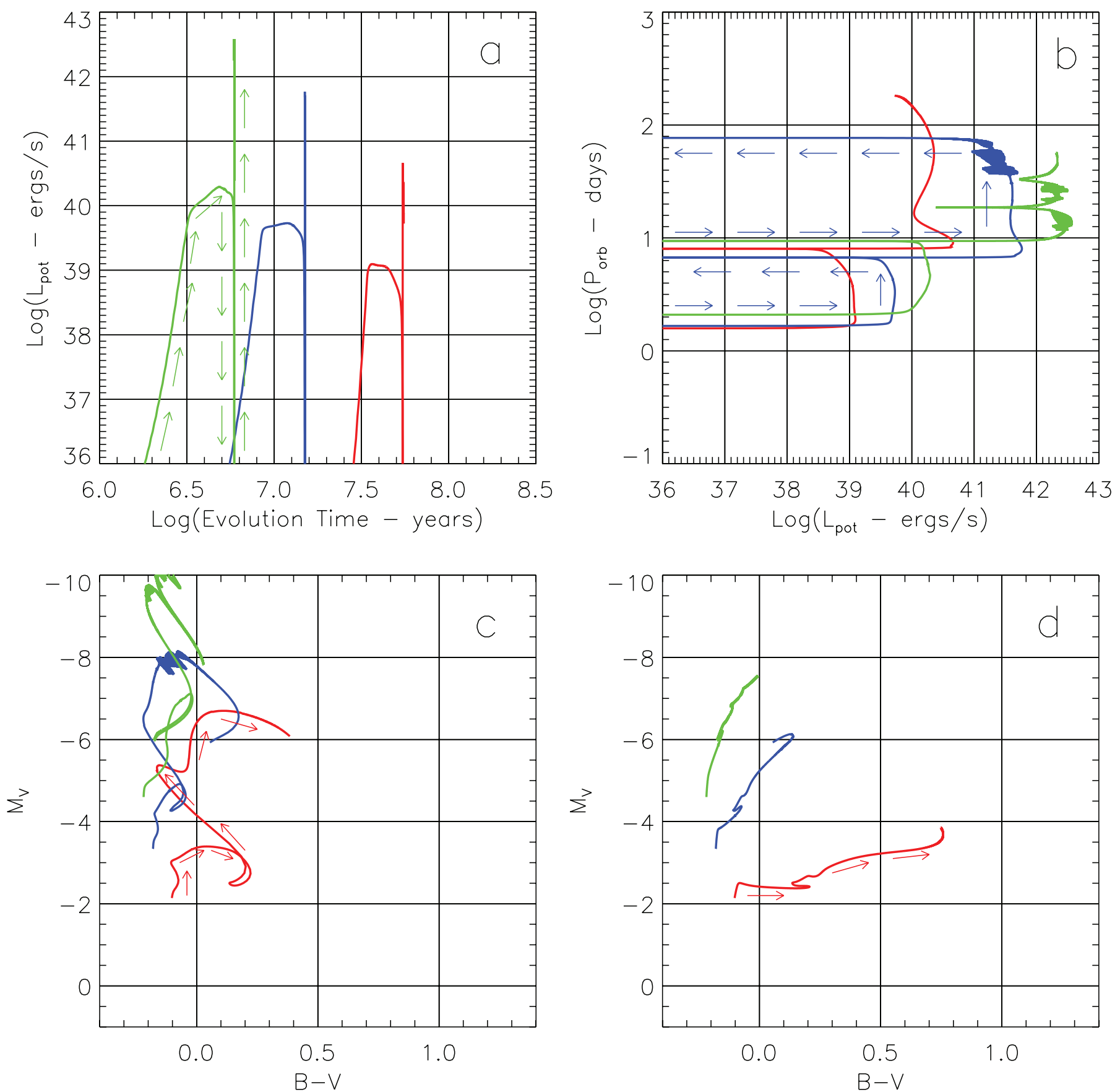

FIg. 3.-Evolutionary tracks of illustrative binary evolution calculations. (a): Evolution of X-ray luminosity with age of the system. (b): Orbital period vs. X-ray luminosity. $(c)$ : CMD for the binary system. $(d)$ : CMD of the donor star alone. The green curves show the evolution of an IMBH binary, typical of model Ic, with an initial donor mass $\left(M_{\mathrm{don}}\right)$ of $30 M_{\odot}$, a black hole mass $\left(M_{\mathrm{BH}}\right)$ of $1000 M_{\odot}$, and an initial orbital period $\left(P_{\mathrm{orb}}\right)$, of 2.1 days. The blue curves represent model Lb with $M_{\mathrm{don}}=14 M_{\odot}$, $M_{\mathrm{BH}}=23 M_{\odot}$, and $P_{\text {orb }}=1.7$ days. And the red curves represent model La with $M_{\mathrm{don}}=7 M_{\odot}, M_{\mathrm{BH}}=11 M_{\odot}$, and $P_{\text {orb }}=1.6$ days.

through the inner Lagrange point. The $L_{\mathrm{pot}}-t_{\mathrm{ev}}$ curve depicts a small increase in $L_{\mathrm{pot}}$ as the donor evolves through the main sequence. For high-mass stars, as considered here, there is an overall contraction phase before the star ascends the giant branch. During the contraction phase, even a small decrease in radius leads to a large reduction in the mass-transfer rate, $\dot{M}$, and hence the observed dip in $L_{\mathrm{pot}}$. After the contraction phase, the star ascends the giant branch expanding through the Hertzsprung gap on a thermal timescale. This increase in radius leads to a spike in $L_{\mathrm{pot}}$. The effects of the various phases of evolution of the donor star also manifest themselves through the other properties of the system.
For example, the development of $P_{\text {orb }}$ through the various evolutionary phases is evident from panel $b$. One can see the sharp increase in the orbital period as the star expands on the giant branch. The relatively smaller variations in $L_{\mathrm{pot}}$ as the period changes indicate the near constant, even if rapid, rate of growth of the donor star. Panels $c$ and $d$ depict the evolution of the optical properties of the system. As is apparent from panel $d$, the increase in the absolute magnitude of the donor star as it ascends the giant branch takes place along with a corresponding decrease in its effective temperature. In the context of a binary system, however, some of the X-ray flux is reprocessed in the accretion disk, 

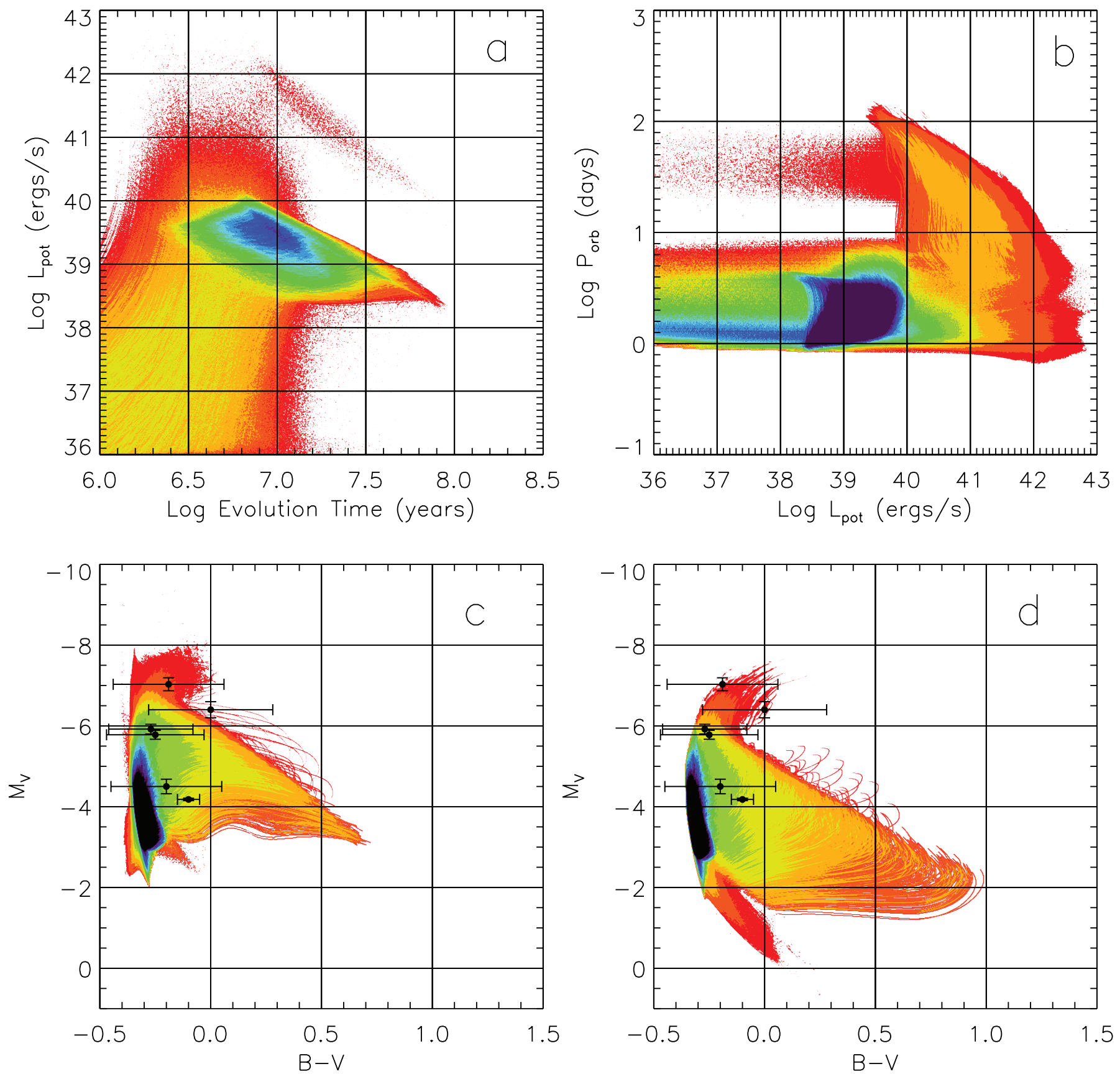

Fig. 4.-Population diagrams for LMBH model La, with $\lambda \mathcal{E}_{\mathrm{CE}}=0.015$ and a maximum primary (i.e., black hole progenitor) mass of $45 M_{\odot}$. (a): Evolution of potential X-ray luminosity with age of the system. (b): Orbital period vs. potential X-ray luminosity. (c): CMD of the binary systems. The irradiation is taken to be from the full $L_{\mathrm{pot}}$. $(d)$ : CMD for donor stars alone. For both $(c)$ and $(d)$, contributions to the CMD image are made only during times when $L_{\mathrm{pot}} \gtrsim 2 \times 10^{39}$ ergs s $\mathrm{s}^{-1}$. Each diagram is an image of $700 \times 700$ pixels and represents the 30,000 X-ray binary evolution calculations that we computed. For each diagram, the parameter values from all the tracks were registered in each pixel that was traversed. The intensity in $(a)$ represents the number of systems in the pixel, whereas the intensity in each of the other panels represents the accumulated evolution time spent in the pixel. The colors in $(a),(b),(c)$, and $(d)$ are scaled according to the $\frac{1}{2}, \frac{1}{3}, \frac{1}{4}$, and $\frac{1}{4}$ roots, respectively, of the actual accumulated values, with purple being of highest intensity and red being the lowest; the actual ratio of values between purple and red is $\sim 200: 1$ in $(a)$ and $\sim 1000: 1$ in $(b)-(d)$. Each distinct change in color corresponds to roughly a factor of $\sim 2$ in actual value in $(a)$ and $(b)$, and a factor of $\sim 3$ in $(c)$ and $(d)$.

making the systems bluer and more luminous, as can be seen from panel $c$. The tracks in panel $c$ also reflect the effects of mass transfer.

\subsection{Population Diagrams}

Figures 4, 5, and 6 show color images of 30,000 evolutionary tracks each, corresponding to models $\mathrm{La}, \mathrm{Lb}$, and Ic, respectively (see Table 2). The panel arrangement and parameters shown are the same for all three figures and match those of Figure 3. Each panel contains a $700 \times 700$ image matrix of the corresponding parameters. For panel $a$, the matrix covers 7 decades in $L_{\mathrm{pot}}$ and
2.5 decades in evolution time, in equally spaced logarithmic intervals. For each step of an evolutionary track, the position of the $L_{\mathrm{pot}}-t_{\mathrm{ev}}$ pair is located in the matrix and a value of 1 is added to the corresponding matrix element. The resulting matrix, after recording all the evolution steps from all the 30,000 tracks, is displayed as a color image, with square-root scaling in intensity to enhance the dynamic range. For panel $b$, the matrix covers 4 decades in $P_{\mathrm{orb}}$ and 7 decades in $L_{\mathrm{pot}}$, in equally spaced logarithmic intervals. Each time the step of an evolution track lands in a matrix element, the evolution time-step is added to the corresponding 

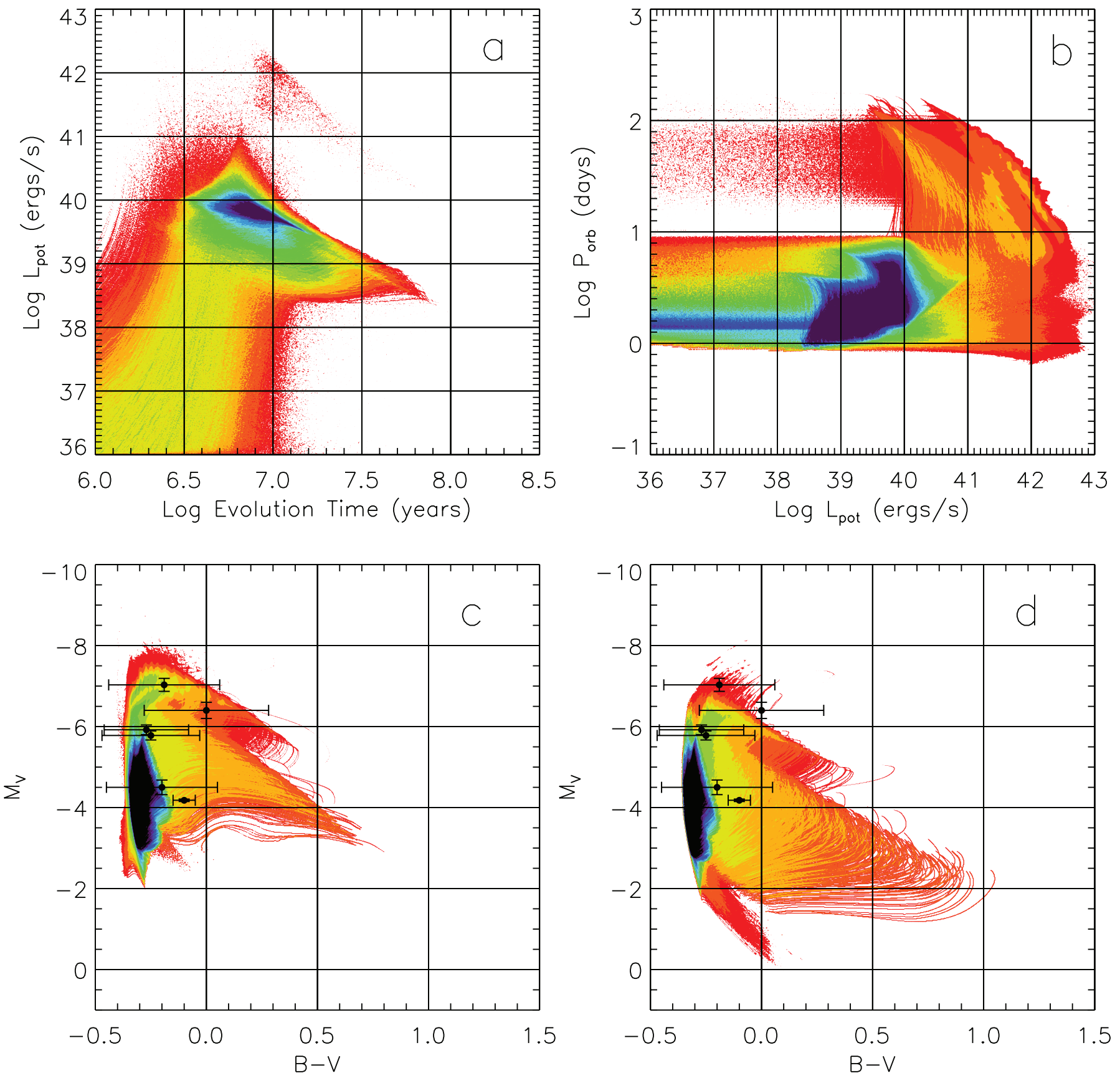

FIG. 5.- Same as Fig. 4, but for $\mathrm{LMBH}$ model Lb, with $\lambda \mathcal{E}_{\mathrm{CE}}=0.015$ and a maximum progenitor primary mass of $65 M_{\odot}$.

matrix element, as opposed to adding a value of 1 as was the case for panel $a$. For panels $c$ and $d$, the matrices cover 11 units in the absolute visual magnitude, $M_{V}$, and 2 units in color index, in equally spaced linear intervals. Similar to the $P_{\text {orb }}$ versus $L_{\mathrm{pot}}$ image in panel $b$, a value equal to the evolution time step is added to the CMD matrix each time the step of an evolution track lands in a particular matrix element. However, it is extremely important to note that contributions to the CMD are made only during times when $L_{\mathrm{x}} \gtrsim 2 \times 10^{39} \mathrm{ergs} \mathrm{s}^{-1}$, i.e., when the source would be a potential ULX.

The matrices in panels $b, c$, and $d$ are also displayed as color images with $\frac{1}{3}-, \frac{1}{4}$, and $\frac{1}{4}$-root scaling in intensity (i.e., relative probability), respectively, to enhance the dynamic range. The colors reflect this scaling, purple being of highest intensity and red being the lowest.

The intensity in panel $a$ is a measure of the number of systems with a particular $L_{\mathrm{pot}}$ found at a particular time in the evolution of the cluster. These values allow one to calculate the number of active ULX systems in the population at any given epoch and to make estimates of the numbers of ULXs of a certain $L_{\mathrm{pot}}$ in typical galaxies. For all the other images, the intensity at a point is a measure of the total duration (time) spent by all the systems in that interval of parameter space. Since this intensity incorporates both the numbers of systems and the amount of time each system spends in that interval of parameter space, it provides a probability map of the corresponding parameter space. For purposes of discussion later, we shall refer to purple and dark blue regions in these diagrams as regions of high probability and to regions in red as those of low probability.

\subsection{Evolutionary Phases of the Donor Stars}

Comparing panel $c$ from Figure 3, to that of Figures 4, 5, and 6 , we see that the regions of high probability correspond to the 

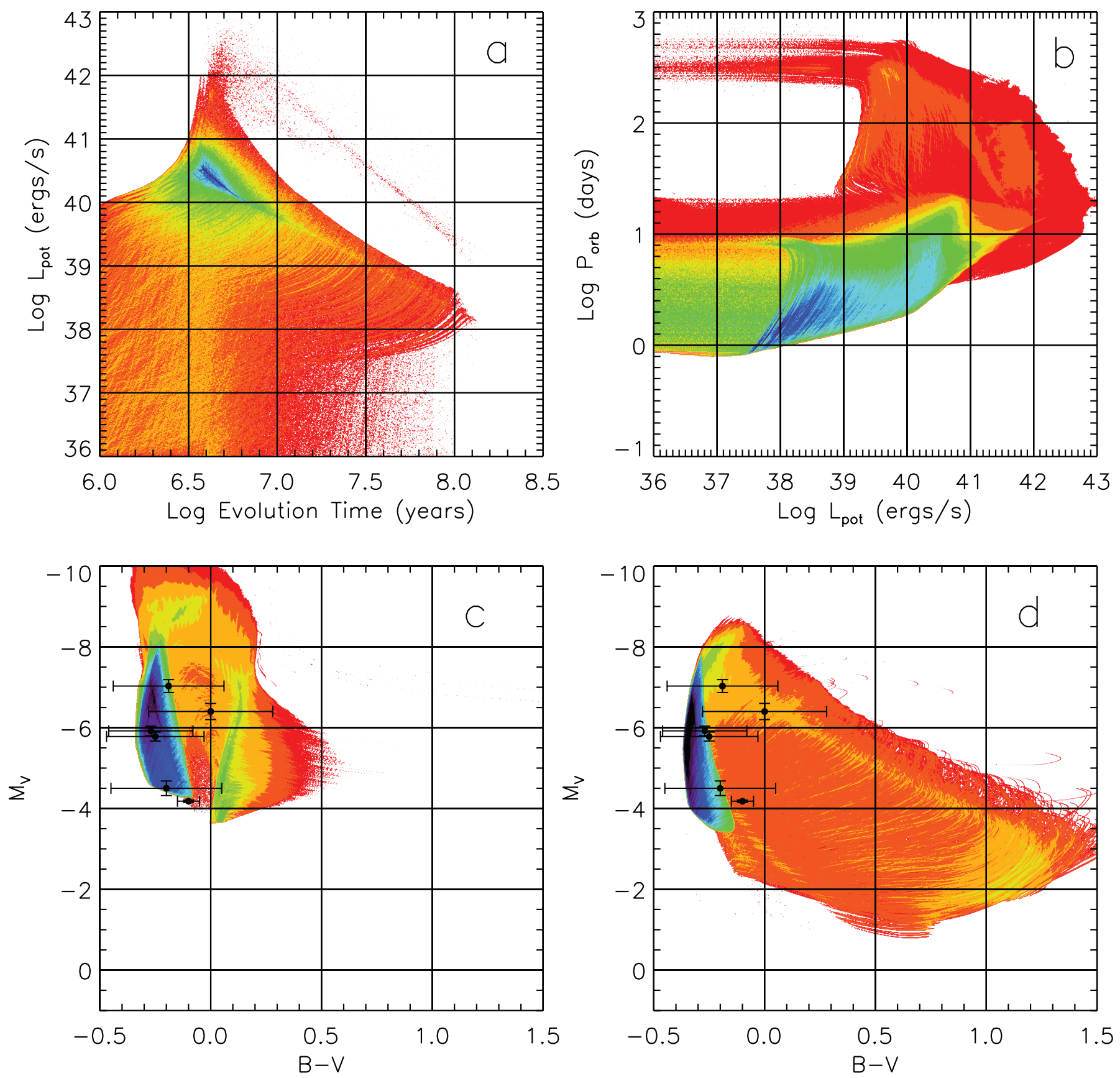

FIG. 6.- Same as Fig. 4, but for IMBH model Ic.

early phases of the donor star's evolution, i.e., on the main sequence or the subgiant branch phase. This is apparent because the donor star spends a predominant amount of its lifetime on the main sequence, and evolves rather quickly on the giant branch. Consequently, the regions of low probability on the diagram are the regions corresponding to the giant-branch phase of the evolutionary tracks or other regions not readily accessed during the binary evolution. However, this description of the phases of evolution with respect to regions on the CMD is only approximate. The exact structure of the CMD is a result of a complex interplay between the evolution of the donor star, its mass loss, and the radiation from the disk at a particular evolutionary stage. From panel $c$ of Figures 4, 5, and 6, we find that, with a high probability, the donor stars belong to the equivalent luminosity classes for single stars of IV and $\mathrm{V}$, with a $B-V$ color range of about -0.35 to -0.10 (corresponding to an effective spectral class of $\mathrm{O}$ through late $\mathrm{B})$.

In many of the panels in Figures 4,5 , and 6 one can discern some of the individual tracks, roughly parallel to each other in the initial stages of the evolution. Tracks higher on the diagram (i.e., lower $M_{V}$ ) correspond to higher mass donors since, at any evolution phase, more massive stars are brighter. Higher mass donor stars become progressively rarer and also spend lesser amounts of time on the main sequence; hence the probability fades toward lower $M_{V}$.

\subsection{Relevance to the Observations}

The data from the optical observations of six ULXs discussed earlier (see Table 1) are plotted along with the model CMDs. For models La (Fig. 4) and Lb (Fig. 5), we see that only three or four 
of the six data points fall on or near high-probability regions of the CMD ( panel $c$ ). However, for the IMBH scenario all six data points fall on or close to the high-probability regions of the CMD in Figure 6. Thus, in the framework of the models presented here, the IMBH scenario appears to be the somewhat more favorable one.

We also see from Figures 4-6 that all the observational CMD points fall in regions corresponding to very high-mass donors. This indicates why the IMBH models fit the data somewhat better than the LMBH models. The brighter magnitudes of the observed systems typically require massive donor stars. And, massive donor stars tend to be associated with more massive accretors. The more massive donor stars, in addition to being bluer and more luminous, evolve faster, thereby yielding higher $\dot{M}$ values. In turn, the higher rates of mass transfer increase the X-ray irradiation of the disk as well as the intrinsic disk radiation. The contribution to the system light from disk radiation can be especially important in the case of IMBHs (see $\S 2.5$ ). In the LMBH scenario, the donor masses are limited by the facts that they must be able to trigger a common-envelope phase with the primary star and eject the common envelope, and that they cannot be more than about twice the mass of the $\mathrm{BH}$ accretor in order for stable Roche-lobe mass transfer to take place. In practice, this limits most of the donor masses to be $\lesssim 25 M_{\odot}$ (see Fig. 1). In the IMBH case, on the other hand, the $\mathrm{BH}$ mass has been set at $1000 M_{\odot}$ and the maximum donor mass we consider is $50 M_{\odot}$ (leading to stable mass transfer). Finally, in this regard, we note that the intrinsic light from an IMBH accretion disk can dominate the light (in intensity and color) even in systems where the donor star is on the giant branch and quite cool (compare panels $c$ and $d$ in Fig. 6), and can yield net colors for the system that are blue.

In light of these results, one might ask what would happen if Nature managed to circumvent the constraints on the evolutionary models imposed by our BPS code, and produced LMBH systems with donor masses up to $\sim 50 M_{\odot}$ and with close orbits. Perhaps then the LMBH models would yield CMDs that as naturally match the observations as do the IMBH models. To test this, we computed another set of LMBH models where the black hole masses are taken directly from model $\mathrm{Lb}$, but the initial donor masses and orbital periods are chosen in an ad hoc manner, i.e., using the same prescription as for the IMBH models - with donor masses up to $50 M_{\odot}$. The results are shown in Figure 7. A comparison of this result with that for model Lb (Fig. 5c) shows that while there is some improvement in the agreement with the observations (by about half a magnitude in brightness), the IMBH CMDs are still a somewhat more natural match. The reason for this is straightforward: the most optically luminous systems with blue colors in Figure 6 are dominated by disk radiation, which is much more important in the IMBH than the LMBH systems.

\subsection{Effects of Radiation from the Accretion Disk}

Panel $d$ of each figure shows the CMD of the donor stars alone. Comparing panels $c$ and $d$ in each figure, we see that the difference is most apparent in the IMBH scenario. Comparing panels $c$ and $d$ in Figure 4, we see that the CMDs differ primarily in regions corresponding to the giant-branch phases of the evolutionary tracks, and remain quite similar over the rest of the diagrams. This observation is similar for Figure 5. And since the giant-branch phases lie in the low probability regions of the diagram, one can say that the optical flux from disk radiation contributes relatively little to the observed optical appearances of ULXs in the LMBH scenario. By contrast, for the IMBH model, a comparison of panels $c$ and $d$ in Figure 6 shows that the contribution of disk irradiation and intrinsic disk radiation from viscous losses (see $\S 2.5$ ) is quite

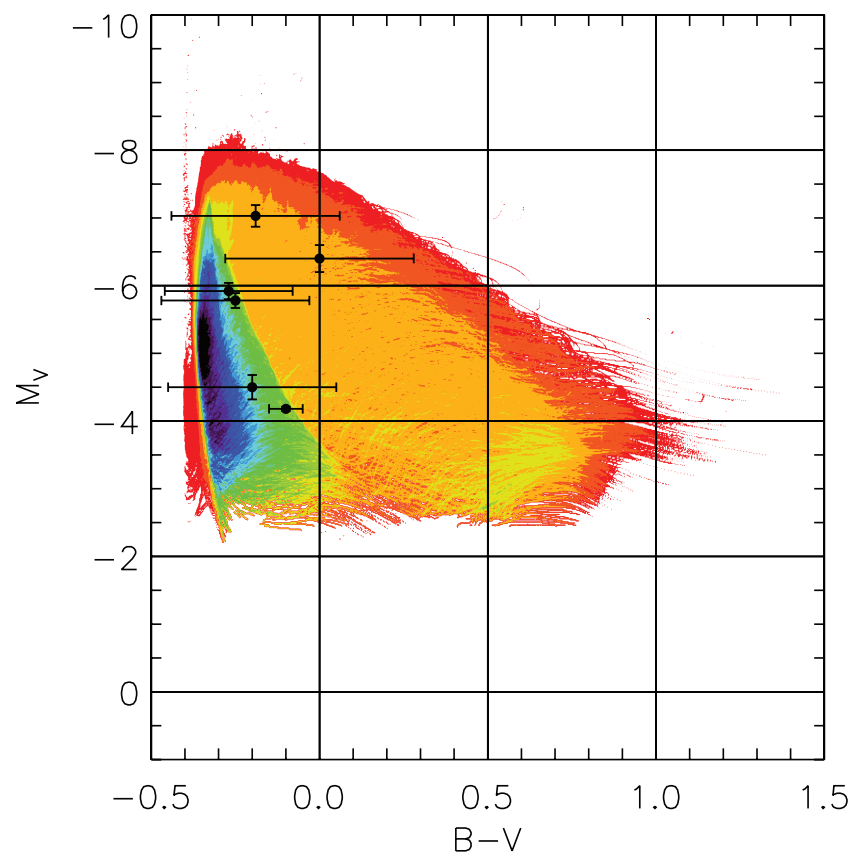

FIG. 7.-CMD distribution for an ad hoc model in which the black hole masses are taken directly from model Lb, but the distributions of initial orbital periods and donor star masses are from model Ic. The magnitudes and colors are for the donor star and the accretion disk combined.

significant in this case, and has substantially altered the CMDs in most phases of the evolution.

The greater influences of the disk radiation for the IMBH model (see Fig. 6) compared to those for the LMBH models seen in Figures 4 and 5 can be explained by two effects. First, in general, the IMBH systems have higher X-ray luminosities by about an order of magnitude-largely by virtue of their higher mass donor stars. This obviously increases the power going into disk irradiation. However, a comparison of IMBH and LMBH systems at the same values of $P_{\text {orb }}$ and $L_{\text {pot }}$ reveals an important difference. The disks in IMBH systems still emit much more optical power than do the LMBH models, and this is predominantly at the blue end of the spectrum. The reason is that there is a substantial amount of intrinsic radiation from the disk (viscous release of gravitational potential energy; see $\S 2.5$ ). The physical explanation for this is straightforward. At the same radial distance from the central black hole, the IMBH system releases $\sim 100$ times more gravitational potential energy than the corresponding LMBH system (simply due to the higher mass of the black hole). This release can end up dominating over the disk irradiation in regions where the bulk of the optical emission is released (see Fig. 2).

Before leaving the subject of disk radiation, we briefly address the issue of whether the irradiation effects in the LMBH models might be greater if the inner parts of the accretion disk were thick, as opposed to the thin-disk assumption we have been adopting. The largest inefficiency factor in the irradiation process (expressed by eq. [4]) is due to the assumption that the inner disk irradiates the outer disk with a Lambertian emission pattern, and that the outer disk lies at a very shallow angle, so that the $\cos \theta$ factor is very small. The possible "benefit" of irradiation from a thick disk might be that the specific X-ray intensity is more strongly peaked in the direction of the outer disk. This effect can be mocked up by utilizing the irradiation expression for central X-ray sources which have hard surfaces (e.g., neutron stars), even though we are not dealing with such accretors. To test the effects of enhancing the $\cos \theta$ factor, we utilized the irradiation expression given by 


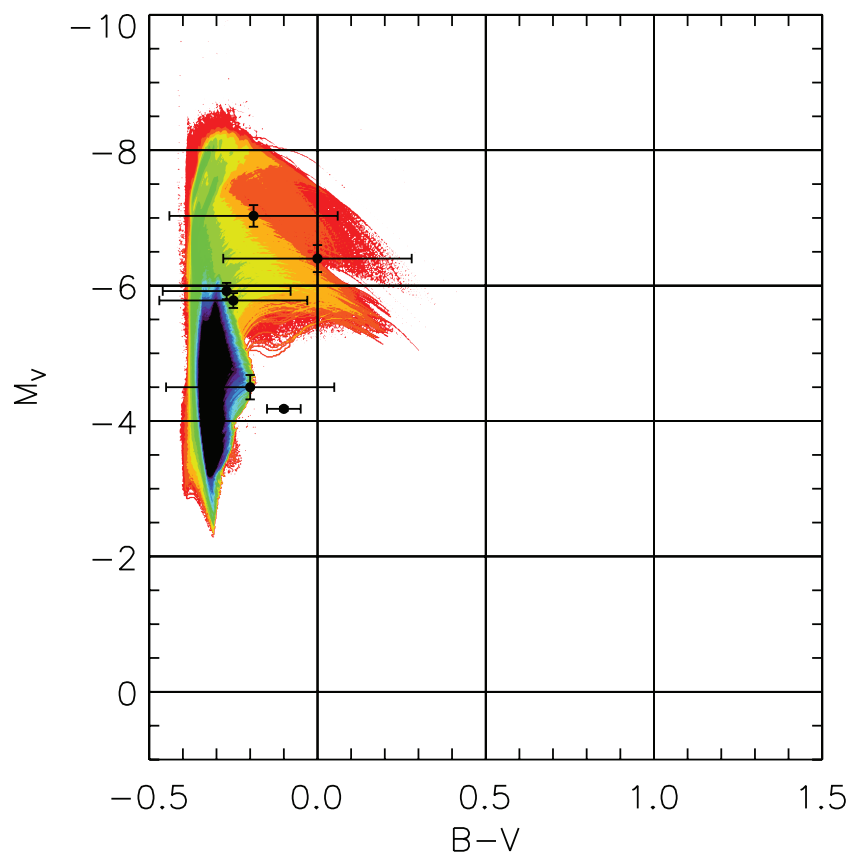

FIG. 8.- CMD distribution for model $\mathrm{Lb}$ which uses an irradiation algorithm that is more efficient in converting X-ray luminosity to optical light in the outer accretion disk. This result is to be compared with that in Fig. $5 c$. The specific irradiation algorithm is given by eq. (8) of Rappaport et al. (2005).

equation (8) in Rappaport et al. (2005). The modified CMD results for model $\mathrm{Lb}$ are shown in Figure 8. This is to be compared with Figure $5 c$. We see that the modified CMD distribution is indeed shifted to higher magnitudes by $\sim 1 \mathrm{mag}$, and systems on the giant branch have been made much bluer; however, the match to the observations is still not quite as good as for model Ic.

Finally, it should be noted that the CMD for the donor star in a binary is expected to be different from that of a single star because of the mass loss via Roche-lobe overflow.

\subsection{Orbital Period versus X-Ray Luminosity}

Panel $b$ in each of Figures 3, 4, 5, and 6 shows the evolution of $L_{\text {pot }}$ with $P_{\text {orb }}$. As can be seen in Figure 3, the variations in $P_{\text {orb }}$ reflect the changes in radius of the donor star through the various phases of evolution. Owing to the dependence of the Roche-lobe radius on the orbital separation, any rapid increase in the radius of the star while in contact with its Roche lobe causes a rapid increase in the orbital separation, and hence in $P_{\text {orb }}$. The final rapid rise in $P_{\text {orb }}$ at the highest $L_{\text {pot }}$ characterizes the expansion of the donor on the giant branch, leading to long orbital periods and high mass-transfer rates. As is apparent, the long values of $P_{\text {orb }}$ in the different models indicate the giant-branch phases of the donors, and the short period regions pertain to the main-sequence and subgiant phases of evolution. The intensity at a given location in panel $b$ is proportional to the number of systems passing through the region and the amount of time spent by the systems in that region. Consequently, the intensity in that region is a measure of the probability of finding a system at that location in parameter space. We then see that, for any model, the most probable regions for $L_{\text {pot }} \gtrsim 10^{39} \mathrm{ergs} \mathrm{s}^{-1}$ lie within a period range of $\sim 1-10$ days.

It follows that the total probability of a system to be in the giant-branch phase is proportional to the sum of all the evolution times recorded in the long-period region, taken to be $P_{\text {orb }} \gtrsim 10$ days. Similarly, the total probability of being in the pregiant phase is proportional to the sum of all the evolution times recorded in the short-period region, i.e., $P_{\text {orb }} \lesssim 10$ days. Thus, we can calculate the relative probability for a ULX donor to be in the giant-branch phase versus that to be on the pregiant branch by calculating the ratios of the two sums for all $L_{\text {pot }} \gtrsim 2 \times 10^{39} \mathrm{ergs} \mathrm{s}^{-1}$. The relative probabilities thus calculated are $0.02,0.01$, and 0.05 for models $\mathrm{La}, \mathrm{Lb}$, and Ic, respectively. This reflects, in a quantitative way, the known fact that the systems are more likely to be found in the pregiant phase than in the giant phase because of the relatively small amount of time they spend on the giant branch.

The implication of the $P_{\text {orb }}-L_{\text {pot }}$ population diagram lies in the fact that given an observed value of $L_{\text {pot }}$, one can estimate the most probable orbital period corresponding to each of the three ULX scenarios presented in Figures 4, 5, and 6. For instance, given an observed $L_{\text {pot }}=10^{40} \mathrm{ergs} \mathrm{s}^{-1}$, we see from Figures 4, 5, and 6 that the most probable periods lie between $1-5,1-5$, and $2-10$ days for the models $\mathrm{La}, \mathrm{Lb}$, and Ic, respectively. Thus, the diagrams serve as rough guidelines showing what orbital periods are to be expected.

\subsection{Evolution of the X-Ray Luminosity}

Panel $a$ of each of Figures 4, 5, and 6 shows a population diagram of the evolution of the X-ray luminosity $\left(L_{\text {pot }}\right)$ with the age of the system. Defining the ULX luminosities as $L_{\text {pot }} \gtrsim 2 \times$ $10^{39} \mathrm{ergs} \mathrm{s}^{-1}$, we see that the systems in model Ic are most active as ULX systems between $\sim 2$ and $6 \mathrm{Myr}$, while systems in models $\mathrm{La}$ and $\mathrm{Lb}$ are most active between $\sim 3$ and 20 Myr. These modest differences in active cluster lifetimes can be explained on the basis of the ranges of donor masses in the different models. Donors with higher masses have shorter nuclear evolution timescales, and hence shorter overall lifetimes. So, the higher the donor masses, the shorter will be the active lifetimes of the systems, leading to shorter overall timescales over which a star cluster containing such binaries is X-ray active. Shorter lifetimes of systems also mean that the mass-transfer rates are higher, leading to systems with higher luminosities. Consequently, while systems in model Ic can produce high $L_{\text {pot }}$ systems even before ascending the giant branch, systems with lower masses have to wait longer to produce their peak X-ray luminosities. It also follows that the numbers of systems having high $L_{\text {pot }}$ will contain a higher proportion of higher-mass donors.

Figure 9 elucidates this latter point. It shows plots of fractions of the initial systems that have $L_{\text {pot }}$ greater than $10^{36}, 2 \times 10^{39}$, and $10^{40} \mathrm{ergs} \mathrm{s}^{-1}$, at any given evolution time. We see that the maximum percentage of systems having (potential) ULX luminosities at any time during the age of a star cluster is $\sim 40 \%$ for model $\mathrm{La}, \sim 60 \%$ for model $\mathrm{Lb}$, and $\sim 25 \%$ for model Ic. We also see that the percentages of systems having $L_{\text {pot }}>10^{40} \mathrm{ergs} \mathrm{s}^{-1}$ are $\sim 3 \%, \sim 15 \%$, and $\sim 20 \%$, respectively, for these same models. Thus we find that the LMBH model, $\mathrm{Lb}$, is competitive with the IMBH model in this regard - provided, of course, that it can be demonstrated that stellar-mass black hole accretors can exceed the Eddington limit by factors of 10 or more.

\subsection{Estimates of ULX Numbers}

Based on the $L_{\text {pot }}-t_{\text {ev }}$ population diagrams, we can also make rough estimates of the numbers of ULXs of different $L_{\text {pot }}$ that can be found in a galaxy in steady state, assuming the different models of ULX formation.

Each element of the $L_{\mathrm{pot}}-t_{\mathrm{ev}}$ matrix contains the number of $\mathrm{BH}$ binary systems that have luminosities between $L_{\text {pot }}$ and $L_{\text {pot }}+$ $\Delta L_{\text {pot }}$ and ages between $t_{\mathrm{ev}}$ and $t_{\mathrm{ev}}+\Delta t_{\mathrm{ev}}$. Both $L_{\mathrm{pot}}$ and $t_{\mathrm{ev}}$ are logarithmically spaced with 100 bins per decade. Given the fact that we evolve 30,000 binary systems for each model, the fraction of all high-mass binary systems that lie in the above mentioned 


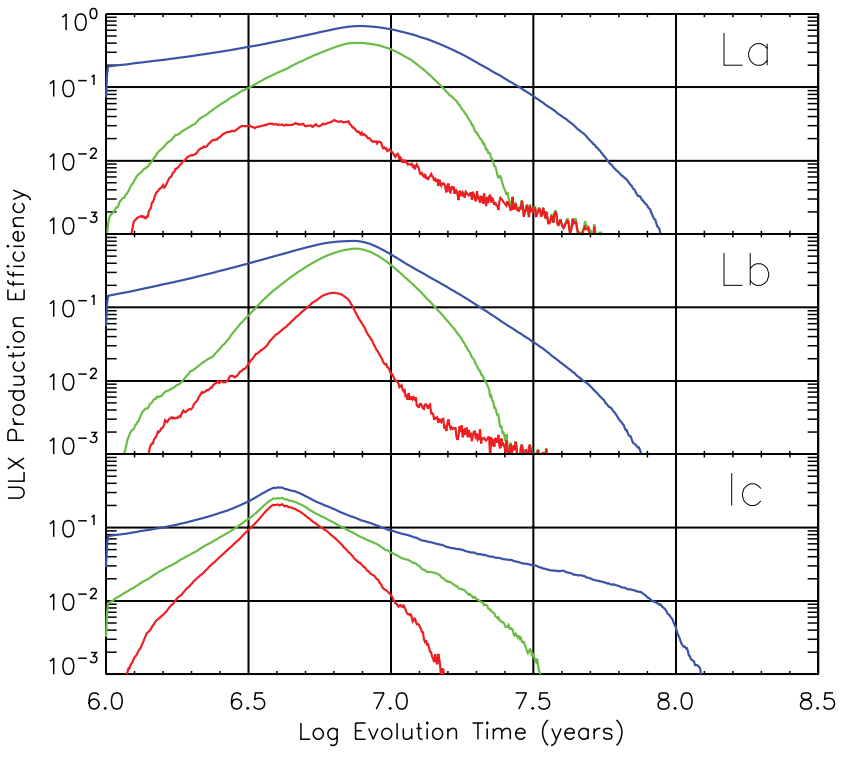

FIG. 9.- Fraction of LMBH/IMBH binaries as a function of star cluster evolution time for three different lower limits on the X-ray luminosity. The different models are labeled in the panels. For each model, the blue curves represent fractions of the initial systems that have $L_{\text {pot }} \gtrsim 10^{36} \mathrm{ergs} \mathrm{s}^{-1}$; the green curves represent fractions with $L_{\text {pot }} \gtrsim 2 \times 10^{39} \mathrm{ergs} \mathrm{s}^{-1}$, i.e., ULX luminosities; and the red curves are for fractions of systems with $L_{\text {pot }} \gtrsim 10^{40} \mathrm{ergs} \mathrm{s}^{-1}$.

intervals of $L_{\mathrm{pot}}$ and $t_{\mathrm{ev}}$ is given by $m\left(L_{\mathrm{pot}}, t_{\mathrm{ev}}\right) / 30,000$, where $m$ denotes the $700 \times 700 L_{\text {pot }}-t_{\mathrm{ev}}$ matrix.

For the LMBH models, the $\mathrm{BH}$ accretors in this age interval, $\Delta t_{\mathrm{ev}}$, have all resulted from core-collapse supernovae ( $\mathrm{SNe}$ ) in an equal time interval at some epoch, $t_{\mathrm{ev}}$, in the past. Assuming a uniform SN core-collapse rate of $R_{\mathrm{SN}}$, the total number of $\mathrm{BH}$ binaries formed in this time interval is given by $R_{\mathrm{SN}} \times f_{\mathrm{BH}} \times \Delta t_{\mathrm{ev}}$, where $f_{\mathrm{BH}}$ is the fraction of all core-collapse supernovae that results in BH binaries with high-mass donors (as determined from the BPS code). It then follows that the number of binary systems with luminosities between $L_{\text {pot }}$ and $L_{\text {pot }}+\Delta L_{\text {pot }}$ and evolutionary ages between $t_{\mathrm{ev}}$ and $t_{\mathrm{ev}}+\Delta t_{\mathrm{ev}}$ is given by

$$
\Delta N\left(L_{\mathrm{pot}, j}, t_{\mathrm{ev}, i}\right)=\frac{R_{\mathrm{SN}} f_{\mathrm{BH}}}{30,000} \Delta t_{\mathrm{ev}, i} m\left(L_{\mathrm{pot}, j}, t_{\mathrm{ev}, i}\right),
$$

where $j$ and $i$ label the matrix element bins of $L_{\text {pot }}$ and $t_{\mathrm{ev}}$, respectively. The total number of sources with $L_{\mathrm{x}}>L_{\text {pot }}$ in a galaxy in steady state can be obtained by summing over all the time bins of $t_{\mathrm{ev}}$, and all the luminosity bins greater than $L_{\mathrm{pot}}$ as

$$
N\left(>L_{\mathrm{pot}, k}\right)=\frac{R_{\mathrm{SN}} f_{\mathrm{BH}}}{30,000} \sum_{j=k}^{700} \sum_{i=1}^{700} \Delta t_{\mathrm{ev}, i} m\left(L_{\mathrm{pot}, j}, t_{\mathrm{ev}, i}\right) .
$$

For illustration, we choose a uniform supernova rate $R_{\mathrm{SN}}=$ $0.01 \mathrm{yr}^{-1}$ (e.g., to represent a typical Milky Way-type galaxy) and $f_{\mathrm{BH}} \simeq 3.4 \times 10^{-6}$ and $4.6 \times 10^{-6}$ for models La and Lb, respectively. These values of $f_{\mathrm{BH}}$ are deduced from the results of our BPS code. The computed steady-state cumulative luminosity functions for models $\mathrm{La}$ and $\mathrm{Lb}$ are shown, respectively, in the red and blue curves of Figure 10.

For the IMBH model, we consider the IMBHs to be formed dynamically in young star clusters. Following a formulation similar to equation (6) for LMBH models, we find for the IMBH model:

$N\left(>L_{\mathrm{pot}, k}\right)=\frac{R_{\mathrm{ysc}} f_{\mathrm{IMBH}} f_{\mathrm{cap}}}{30,000} \sum_{j=k}^{700} \sum_{i=1}^{700} \Delta t_{\mathrm{ev}, i} m\left(L_{\mathrm{pot}, j}, t_{\mathrm{ev}, i}\right)$,

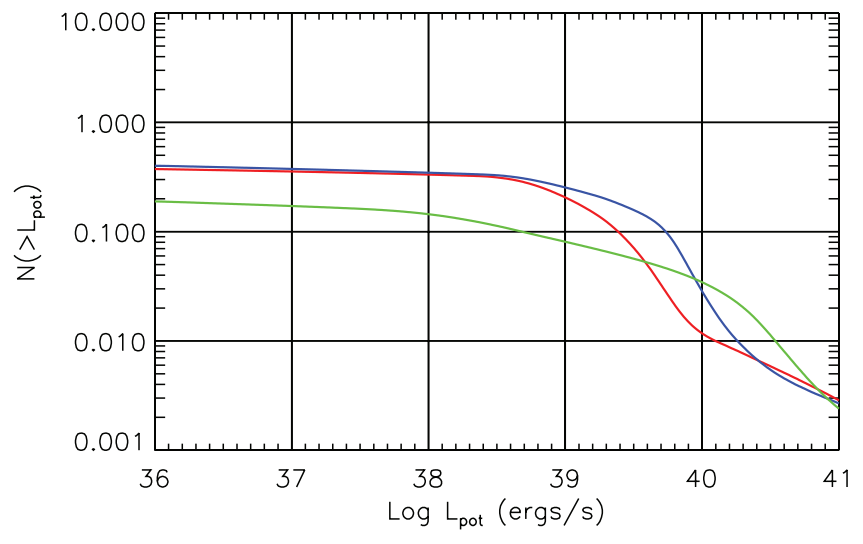

FIG. 10.-Expected numbers of ULXs at the present epoch as a function of $\mathrm{X}$-ray luminosity in a galaxy with a core-collapse supernova rate of $0.01 \mathrm{yr}^{-1}$. The red, blue, and green curves correspond to models La, Lb and Ic, respectively.

where $R_{\text {ysc }}$ is the formation rate of young star clusters (with $M_{\text {clus }} \gtrsim 10^{4} M_{\odot}$ ) in a typical spiral galaxy, $f_{\mathrm{IMBH}}$ is the fraction of all such clusters that form an IMBH, and $f_{\text {cap }}$ is the fraction of all such IMBHs that capture a massive companion into a close orbit. As illustrative values, we choose a uniform $R_{\mathrm{ysc}}=10^{-5} \mathrm{yr}^{-1}$ (estimated from the combined work of de Grijs et al. 2003; Hunter et al. 2003; Grimm et al. 2003), $f_{\mathrm{IMBH}} \simeq 0.1$, and $f_{\text {cap }} \simeq 0.05$ (see, e.g., Blecha et al. 2006). The distribution of the numbers of systems as a function of $L_{\text {pot }}$ is shown by the green curve in Figure 10.

It can be seen from Figure 10 that the expected numbers of ULX systems per galaxy are in fair agreement with the observations (Grimm et al. 2003; Ptak \& Colbert 2004) for the IMBH model, albeit with very large uncertainties. Specifically, the estimates in Figure 10 indicate that a typical galaxy is expected to harbor $\sim 0.07$ and $\sim 0.03$ IMBH ULXs with $L_{\text {pot }}>2 \times 10^{39}$ and $10^{40} \mathrm{ergs} \mathrm{s}^{-1}$, respectively. For the LMBH models, the expected numbers range from about $\sim 0.1-0.2$ for $L_{\text {pot }}>2 \times 10^{39} \mathrm{ergs} \mathrm{s}^{-1}$ per galaxy to $\sim 0.01-0.03$ with $L_{\text {pot }}>10^{40} \mathrm{ergs} \mathrm{s}^{-1}$. These LMBH model numbers are reasonably matched with the observations (Grimm et al. 2003; Ptak \& Colbert 2004).

\section{SUMMARY AND CONCLUSIONS}

In this paper we have explored the optical properties and other observable parameters for a range of ULX models. We investigated a very large number of systems from three different ULX populations, two consisting of stellar-mass $\mathrm{BH}$ accretors, which we refer to as stellar-mass (i.e., low-mass) black holes (LMBHs) and one consisting of intermediate-mass black hole accretors (IMBHs). For each population, we computed the evolution of 30,000 individual binary systems and generated population diagrams to explore a wide region of parameter space. We computed optical CMDs for the donor stars and for the binary systems including radiation from the accretion disk. We also computed population diagrams showing the evolution of $L_{\text {pot }}$ with donor age, and those showing the evolution of $L_{\text {pot }}$ with $P_{\text {orb }}$.

When we plot the observed colors and magnitudes for six ULX systems found in the literature on the model CMDs, we find that all the data points lie in or near the high-probability regions spanned by the IMBH models on the CMD. On the other hand, only three or four of the observed systems lie close to the highprobability region spanned by the LMBH models La and Lb, respectively. In light of this, we conclude that IMBH models are somewhat favored by the optical observations. The locations of the observational data points on the CMDs indicate that these systems correspond to high-mass donors, with $M_{\text {don }} \gtrsim 25 M_{\odot}$. 
The more massive donor stars are bluer and more luminous, evolve faster, and produce higher $\dot{M}$ values. In turn, the higher rates of mass transfer increase the disk radiation (both intrinsic and due to irradiation), especially in the IMBH systems ( $\operatorname{see} \oint \S 2.5,3.4$, and 3.5). This provides an indication as to why the IMBH models are somewhat favored over LMBH models. Our BPS calculations for LMBH binaries do not produce very many successful systems with a donor mass of $\gtrsim 25 M_{\odot}$, owing to limitations on the mass of the $\mathrm{BH}$ ( $\lesssim 15$ and $24 M_{\odot}$ for models La and $\mathrm{Lb}$, respectively) and the requirement of stable mass transfer (but see $\S 3.4$ and Fig. 7). On the other hand, the mass of the $\mathrm{BH}$ accretor in the IMBH models is set at $1000 M_{\odot}$, allowing for very massive donor stars, and hence the ability to account for higher optical luminosities. Finally, in this regard, we note that the intrinsic light from the IMBH accretion disk can dominate the system light (in intensity and color), thereby keeping these systems luminous and blue even when the donor star is on the giant branch.

We also find from the CMDs that the regions of high probability correspond to the initial phases of evolution of the donors. This is expected because all stars spend a predominant fraction of their lifetimes on the main sequence. The observational data points all lie in or very near the high-probability regions of the IMBH models, and most lie at least near the high-probability regions of the LMBH models. This leads us to conclude that the donor stars in ULXs should be predominantly on the main sequence or on the subgiant branch. The $B-V$ color range of about -0.35 to -0.10 , corresponding to the high-probability regions on the CMDs, indicates that the effective spectral class of the donors should be $\mathrm{O}$ through late $\mathrm{B}$. We also discuss the effects of $\mathrm{X}$-ray irradiation of the accretion disk, as well as intrinsic radiation generated viscously in the disk, on the different models. Disk radiation is found to be significant in the IMBH models, but less so in the LMBH models. We suggest that the main effects operating here are (1) the higher donor masses in the IMBH systems, and hence higher values of $L_{\text {pot }}$, and (2) the larger gravitational potential energy released at a given radial distance in the accretion disk in the IMBH models.

We compute population diagrams showing the evolution of $L_{\text {pot }}$ with $P_{\text {orb }}$. As concluded previously from the CMDs, the $P_{\text {orb }}-L_{\text {pot }}$ diagrams show that the giant branch phases of evolution in these systems form a low-probability region in parameter space. It is seen that the most probable orbital periods for all the models lie in the range of $\sim 1-10$ days, corresponding to the main-sequence and subgiant phases of evolution. We quantify the likelihood of ULXs being in the pregiant phase by estimating the relative probabilities for the systems to be in a pregiant phase versus the giant phase. We find the probabilities to be in the ratios $0.02,0.01$, and 0.05 for models $\mathrm{La}, \mathrm{Lb}$ and Ic, respectively. We subsequently discuss the utility of the $P_{\text {orb }}-L_{\text {pot }}$ diagrams for estimating the probable range of $P_{\text {orb }}$ for a system with a known $L_{\text {pot }}$.

Finally, we compute population diagrams showing the evolution of $L_{\text {pot }}$ with the evolution time of the host star cluster. Similar diagrams have been reported for various ULX models in our previous studies (see Rappaport et al. 2005; Madhusudhan et al. 2006). In this paper, we use the diagrams to calculate the ULX production efficiency as a function of the age of a star cluster, for the different models. We also estimate the numbers of ULXs in steady state for a typical galaxy as a function of $L_{\text {pot }}$. We find, with crude estimates, that the IMBH models explain the observed numbers of ULXs reasonably well, with $\sim 0.07$ with $L_{\text {pot }}>2 \times$ $10^{39} \mathrm{ergs} \mathrm{s}^{-1}$ per Milky Way-type galaxy to $\sim 0.03$ per galaxy with $L_{\text {pot }}>10^{40} \mathrm{ergs} \mathrm{s}^{-1}$. For the LMBH models, the numbers range from about $\sim 0.1-0.2$ for $L_{\text {pot }}>2 \times 10^{39} \mathrm{ergs} \mathrm{s}^{-1}$ per galaxy to $\sim 0.01-0.03$ with $L_{\text {pot }}>10^{40} \mathrm{ergs} \mathrm{s}^{-1}$. As discussed in
$\S 3.8$ these numbers are in fair agreement with the observations (Grimm et al. 2003; Ptak \& Colbert 2004). Of course, it should be noted that the production rates of the LMXB systems are very sensitive to the (uncertain) choice of the common-envelope parameters $\mathcal{E}_{\mathrm{CE}} \times \lambda$. Likewise, the production rates for the IMBH systems are highly uncertain because of the poorly understood formation mechanism for IMBHs and the problems associated with capturing the requisite numbers of massive stars into the appropriate orbits with the IMBHs.

With regard to the production rates of ULXs in the LMBH scenario, we should also mention the possibility that for case B mass transfer (i.e., when the primary progenitors of the black holes are in the Hertzsprung gap during the time when mass transfer commences) the primary is more likely to produce a neutron star, rather than a black hole, even for fairly high initial masses (e.g., 50-60 $\left.M_{\odot}\right)$. As has first been argued by Brown et al. (1999) and then has been confirmed in detailed calculations by Brown et al. (2001), this has to do with differences during helium core burning. Basically the final structure of a star is very different depending on whether the star burns helium with a hydrogen-burning shell around it or without the hydrogen-burning shell (as one would expect if the star loses its envelope before or early during helium burning). Without the hydrogen-burning shell the star ends up with a much smaller iron core at the end and most likely results in the production of a neutron star. This could somewhat reduce the number of predicted LMBH ULXs.

As an important addendum to the discussion of the LMBH models, we note that there is another possible formation channel for LMBHs that does not involve a common-envelope phase. In this case, the mass ratio of the primordial primary to its secondary is not sufficiently high for a common envelope to form, and the mass transfer may be quasi-stable. In order for the primary to have a well-developed core, it must be past the TAMS, implying typical orbital periods $\gtrsim 10$ days when mass transfer commences. The behavior of the subsequent mass transfer onto the secondary, i.e., the fraction of the matter that is retained and the specific angular momentum carried away by matter lost from the system, is highly uncertain. The orbit may widen or possibly shrink, but perhaps is most likely to remain relatively unchanged from the onset to the end of the mass-transfer phase. As the exposed core evolves toward collapse, any matter lost in a stellar wind mostly serves to widen the orbit still further. Our preliminary BPS calculations of such systems indicate that they may be formed at a much higher rate than those LMBHs that go through a common-envelope phase, as considered in this work. However, once the secondary star (now the donor) evolves and starts to transfer mass to the black hole, $\dot{M}$ can be extremely large (due to the fact that the donor is substantially evolved) and the mass-transfer phase is quite short lived, i.e., of order of a few thousand years. Our preliminary binary evolution study of these systems shows that the combination of high formation rates and short mass-transfer phases may yield populations of potential ULX systems, formed without common envelopes, that are competitive with those we have considered in this work. The parameter space (e.g., mass capture fractions and specific angular momentum losses) that would need to be studied in order to evaluate the populations of such systems is sufficiently great that an entire study should be devoted to this formation channel. We plan to explore this in future work.

In estimating the numbers of ULXs, we have allowed for violation of the Eddington limit by using $L_{\mathrm{pot}}$ in our calculations. This relaxation is imperative in order to explain ULXs with $L_{\mathrm{x}} \gtrsim$ $10^{40} \mathrm{ergs} \mathrm{s}^{-1}$ using the LMBH models. However, the scenario by which the Eddington limit could be violated in a $\mathrm{BH}$ binary is not clear. We note that the numbers for the LMBH models with 
$L_{\text {pot }} \gtrsim 10^{40} \mathrm{ergs} \mathrm{s}^{-1}$ could be quite unphysical because, for these luminosities, $L_{\text {pot }} \gg L_{\text {Edd }}$. The Eddington limit for a $20 M_{\odot}$ accretor is $\sim 2.5 \times 10^{39} \mathrm{ergs} \mathrm{s}^{-1}$. Allowing for the fact that a massive donor star could be transferring helium onto the accretor during the later stages of its evolution, the Eddington limit is increased by a factor of 2 , to $\sim 5 \times 10^{39} \mathrm{ergs} \mathrm{s}^{-1}$. Considering, however, that the bolometric luminosity may well be a factor of $\sim 2-3$ times the $\mathrm{X}$-ray luminosities observed in the 1-10 keV X-ray band, the effective Eddington limit in the X-ray band is only $\sim 1.5 \times 10^{39} \mathrm{ergs} \mathrm{s}^{-1}$. Thus, even if we allow for a violation of the Eddington limit by reasonable factors of a few, and thereby achieve $L_{\text {pot }}$ up to $\sim 1.5 \times$ $10^{40} \mathrm{ergs} \mathrm{s}^{-1}$, it is difficult to understand X-ray-band luminosities above $\sim 5 \times 10^{39} \mathrm{ergs} \mathrm{s}^{-1}$.

As for the IMBH model, we have not studied in any detail the formation scenarios of IMBHs or of the capture of binary companions by the IMBHs (but see Portegies Zwart et al. 2004; Tutukov \& Fedorova 2005; Blecha et al. 2006). Despite much evidence in the literature supporting IMBH binaries as candidates for ULXs, the formation mechanisms of IMBHs are not clear. In particular, it is unclear how supermassive stars evolve, and whether they can undergo core collapse to form an IMBH. In calculating the numbers of ULXs in this scenario, we have assumed plausible values for the formation rate of young star clusters in a galaxy, the fraction of these clusters that successfully produce IMBHs, and the probability of capture of a massive companion by the IMBH.

Finally, we should keep in mind the possibility that neither the IMBH nor LMBH binary models may be correct in explaining the most luminous ULXs. For example, it is conceivable that the most luminous ULXs may, in fact, be free-floating IMBHs that are fed by tidally disrupted passing field stars (see Volonteri \& Perna 2005 for a related scenario).

We are grateful to Bill Paxton for all his assistance with the EZ stellar evolution code and to Wlodek Kluźniak for helpful discussions regarding accretion disks. We would also like to thank the referee for very productive comments and suggestions for improving the manuscript. S. R. acknowledges support from NASA Chandra TM5-6003X. L. N. thanks NSERC (Canada) and the CRC Program for financial support, and also acknowledges the CCS at the Université de Sherbrooke for technical assistance.

\section{REFERENCES}

Abramowicz, M. A., Czerny, B., Lasota, J. P., \& Szuszkiewicz, E. 1988, ApJ, 332,646

Bardeen, J. M. 1970, Nature, 226, 64

Begelman, M. 2002, ApJ, 568, L97 2006, ApJ, 643, 1065

Belczynski, K., Taam, R. E., Kalogera, V., Rasio, F. A., \& Bulik, T. 2007, ApJ, 662, 504

Blecha, L., Ivanova, N., Kalogera, V., Belczynski, K., Fregeau, J., \& Rasio, F. 2006, ApJ, 642, 427

Brown, G. E., Heger, A., Langer, N., Lee, C.-H., Wellstein, S., \& Bethe, H. A. 2001, NewA, 6, 457

Brown, G. E., Lee, C.-H., \& Bethe, H. A. 1999, NewA, 4, 313

Colbert, E., \& Mushotzky, R. 1999, ApJ, 519, 89

Copperwheat, C., Cropper, M., Soria, R., \& Wu, K. 2005, MNRAS, 362, 79

2007, MNRAS, 376, 1407

Cropper, M., Soria, R., Mushotzky, R. F., Wu, K., Markwardt, C. B., \& Pakull, M. 2004, MNRAS, 349, 39

de Grijs, R., Anders, P., Bastian, N., Lynds, R., Lamers, H. J. G. L. M., \& O’Neil, Jr., E. J. 2003, MNRAS, 343, 1285

Dewi, J., \& Tauris, T. 2000, A\&A, 360, 1043

Ebisawa, K., Życki, P., Kubota, A., Mizuno, T., Mushotzky, R., \& Watarai, K. Y. 2003, ApJ, 597, 780

Eggleton, P. P. 1983, ApJ, 268, 368

Feng, H., \& Kaaret, P. 2007, ApJ, 660, L113

Grimm, H.-J., Gilfanov, M., \& Sunyaev, R. 2003, MNRAS, 339, 793

Han, Z., Podsiadlowski, Ph., Maxted, P. F. L., Marsh, T. R., \& Ivanova, N. 2002, MNRAS, 336, 449

Han, Z., Tout, C. A., \& Eggleton, P. P. 2000, MNRAS, 319, 215

Hopman, C., Portegies Zwart, S. F., \& Alexander, T. 2004, ApJ, 604, L101

Hunter, D. A., Elmegreen, B. G., Dupuy, T. J., \& Mortonson, M. 2003, AJ, 126, 1836

Jaroszyński, M., Abramowicz, M. A., Paczyński, B. 1980, Acta Astron., 30, 1 Johnson, H. L. 1966, ARA\&A, 4, 193

Kaaret, P., Corbel, S., Prestwich, A. H., \& Zezas, A. 2003, Science, 299, 365

Kaaret, P., Ward, M. J., \& Zezas, A. 2004, MNRAS, 351, L83

King, A. R., Davies, M. B., Ward, M. J., Fabbiano, G., \& Elvis, M. 2001, ApJ, 552, L109

King, A. R., Kolb, U., \& Szuszkiewicz, E. 1997, ApJ, 488, 89

Körding, E., Falcke, H., \& Markoff, S. 2002, A\&A, 382, L13

Kuntz, K. D., Gruendl, R. A., Chu, Y.-H., Chen, C.-H. Rosie, Still, M., Mukai, K., \& Mushotzky, R. F. 2005, ApJ, 620, L31

Lang, C., Kaaret, P., Corbel, S., \& Mercer, A. 2007, ApJ, 666, 79

Liu, J-F., Bregman, J. N., \& Seitzer, P. 2002, ApJ, 580, L31

Madhusudhan, N., Justham, S., Nelson, L., Paxton, B., Pfahl, E., Podsiadlowski, Ph., \& Rappaport, S. 2006, ApJ, 640, 918

Maund, J. R., Smartt, S. J., Kudritzki, R. P., Podsiadlowski, Ph., \& Gilmore, G. F. 2004, Nature, 427, 129

Miller, J. M., Fabian, A. C., \& Miller, M. C. 2004, ApJ, 607, 931

Miller, J. M., Fabbiano, G., Miller, M. C., \& Fabian, A. C. 2003, ApJ, 585, L37

Mucciarelli, P., Zampieri, L., Falomo, R., Turolla, R., \& Treves, A. 2005, ApJ, 633, L101

Mucciarelli, P., Zampieri, L., Treves, A., Turolla, R., \& Falomo, R. 2007, ApJ, 658, 999

Pakull, M., \& Mirioni, L. 2003, Rev. Mex. AA Conf. Ser., 15, 197

Paxton, B. 2004, PASP, 116, 699

Pfahl, E. 2005, ApJ, 626, 849

Pfahl, E., Rappaport, S., \& Podsiadlowski, Ph. 2003, ApJ, 597, 1036

Podsiadlowski, Ph., Rappaport, S., \& Han, Z. 2003, MNRAS, 341, 385

Podsiadlowski, Ph., Rappaport, S., \& Pfahl, E. D. 2002, ApJ, 565, 1107

Pols, O. R. 1994, A\&A, 290, 119

Pols, O. R., Tout, C. A., Eggleton, P. P., \& Han, Zh. 1995, MNRAS, 274, 964

Pooley, D., \& Rappaport, S. 2005, ApJ, 634, L85

Portegies Zwart, S. F., Dewi, J., \& Maccarone, T. 2004, MNRAS, 355, 413

Prestwich, A. H., et al. 2007, ApJ, 669, 21

Ptak, A., \& Colbert, E. 2004, ApJ, 606, 291

Rappaport, S., Podsiadlowski, Ph., \& Pfahl, E. 2005, MNRAS, 356, 401

Shakura, N. I., \& Sunyaev, R. A. 1973, A\&A, 24, 337

Socrates, A., \& Davis, S. W. 2006, ApJ, 651, 1049

Soria, R., Cropper, M., Pakull, M., Mushotzky, R., \& Wu, K. 2005, MNRAS, 356,12

Strohmayer, T. E., \& Mushotzky, R. F. 2003, ApJ, 586, L61

Tutukov, A. V., \& Fedorova, A. V. 2005, Astron. Rep., 49, 89

Volonteri, M., \& Perna, R. 2005, MNRAS, 358, 913

Webbink, R. 1985, in Interacting Binary Stars, ed. J. E. Pringle \& R. A. Wade (Cambridge: Cambridge Univ. Press), 39

Wellstein, S., Langer, N., \& Braun, H. 2001, A\&A, 369, 939 